\title{
Accounting for temperature-dependent properties in viscoelastic duct flows
}

\author{
J.M. Nóbrega ${ }^{\text {a }}$, F.T. Pinho ${ }^{\text {b,* }}$, P.J. Oliveira ${ }^{\text {c }}$, O.S. Carneiro ${ }^{\text {a }}$ \\ a Department of Polymer Engineering, IPC-Institute for Polymers and Composites, Universidade do Minho, \\ 4800-058 Guimarães, Portugal \\ b Centro de Estudos de Fenómenos de Transporte, DEMEGI, Faculdade de Engenharia, Universidade do Porto, \\ Rua Dr. Roberto Frias, 4200-465 Porto, Portugal \\ ${ }^{\mathrm{c}}$ Departamento de Engenharia Electromecânica, Universidade da Beira Interior, Rua Marquês, d'Ávila e Bolama, \\ 6201-001 Covilhã, Portugal
}

Received 1 July 2003; received in revised form 3 October 2003

\begin{abstract}
A numerical and theoretical study is conducted to evaluate the effect of temperature-dependent properties on the hydrodynamic and thermal characteristics of viscoelastic fluid flow. The rheological constitutive equation of the fluid under consideration follows a common form of the PTT model, which embodies both influences of elasticity and shearthinning in viscosity. A large number of simulations were carried out for a developing channel flow with an imposed constant wall temperature by varying the parameters controlling elasticity (Weissenberg number) and viscous dissipation (Brinkman number). The resulting Nusselt number and friction factor were determined from the numerical results, for both conditions of constant and temperature-dependent properties. The properties that were allowed to vary with temperature were the viscosity, thermal conductivity, specific heat and relaxation time of the PTT model. From the results it was possible to determine how the usual correlations for $N u$ and $C_{\mathrm{f}}$ have to be modified, following the property-correction method, in order to represent variable-property flow of this viscoelastic fluid. An alternative method to account for variable properties, based on the definition of an equivalent temperature to be used with the constant property $\mathrm{Nu}$ and $C_{\mathrm{f}}$ expressions, is also proposed and shown to be less sensitive to the influence of viscous dissipation. The corrections are highly non-linear and strongly depend on $\varepsilon W e^{2}$ and $B r$ especially when viscous dissipation is weak.
\end{abstract}

(C) 2003 Elsevier Ltd. All rights reserved.

Keywords: Variable viscosity; Temperature-dependent properties; Viscoelastic flows; PTT model

\section{Introduction}

Process engineering and the manufacture of modern consumption items almost always involves non-isothermal flows, and the optimization of these processes requires the ability to predict their characteristics under real flow conditions. For instance, temperature-depen-

\footnotetext{
${ }^{*}$ Corresponding author.

E-mail addresses: mnobrega@dep.uminho.pt (J.M. Nóbrega),fpinho@fe.up.pt (F.T. Pinho),pjpo@ubi.pt (P.J. Oliveira), olgasc@dep.uminho.pt (O.S. Carneiro).
}

dent transport properties, viscous dissipation or other internal sources of energy are invariably present and must be taken into account in the analysis, additionally the flow configuration and the flow itself may not be geometrically simple. In most instances, such features preclude analytical solutions of the coupled hydrodynamic, thermal energy and constitutive equations, and recourse to numerical methods becomes mandatory.

With the non-Newtonian fluids that are usually found in such relevant processes as in the chemical, the food, the paint or the plastics industries, to name but a few, complications are enhanced by the non-linearity of 
the constitutive equation and it is not surprising to see major efforts at developing adequate numerical methods especially for handling flows of such complex rheological behaviour fluids. These materials are usually rather viscous, require high temperatures for attaining the liquid state, as in the case of polymer melts, and large temperature differences may also be present. These conditions lead to effects such as internal heat generation by viscous dissipation and, consequently, a temperature-dependent viscosity must be considered. Both factors have a significant impact [1] on the flow and heat transfer characteristics. Other transport and thermodynamic properties, such as thermal conductivity, density and specific heat, have a weak dependence on temperature so that the assumption of their temperature invariance has less serious consequences [2], as will be seen later.

The relevance of these factors on flow and heat transfer performance has long been recognised, but early works have focused on simple fluids and geometries to gain insight into the problem. The issue of temperaturedependent properties is not restricted to non-Newtonian fluids; in the quest for ever more efficient processes, analysis of laminar flows of Newtonian fluids with temperature-dependent viscosity have recently being carried out to help understand and develop mechanisms of heat transfer enhancement and its relationship with buoyancy [3]; such efforts can be traced back at least to the mid twentieth century, as an inspection of the reference list of Chou and Tung [3] shows (for instance, the 1951 NACA report of Deissler). Textbooks like Kays and Crawford [4] or Eckert and Drake [5] explain in detail the treatment of high velocity convection for Newtonian fluids, and suggest methods for correcting the correlations derived on the basis of constant fluid properties to account for their temperature dependence and also for the effect of viscous dissipation. The methods they present for Newtonian fluids have been extended also to non-Newtonian inelastic fluids, as in some of the works referred in the remaining paragraphs of this introduction.

Using several generalised Newtonian models, such as power law, Ellis or Prandtl-Eyring fluids, several authors have investigated the effects of viscous dissipation and/or temperature-dependent viscosity on fully-developed and developing pipe flow. A comprehensive summary of such developments is presented by Forrest and Wilkinson [6] for laminar pipe flow of power law fluids, and by Skelland [7] for other duct flows and fluids. For other more complex flows of industrial relevance the solution of the equations becomes much more complex and can only be achieved numerically, unless simplifying assumptions are made, as in Kenny et al. [8],who investigated the extrusion of thermoplastics including the effect of slip at the walls, or in the rectangular duct work of Shin and Cho [9].
Investigations with viscoelastic fluids, where the effects of temperature-dependence of the viscosity are accounted for, are much scarcer, but no less relevant. A major limitation is the convergence difficulty found with iterative numerical methods for highly elastic flows, and so the attention of researchers has been mostly concentrated on resolving this issue. Nonetheless, we identify the numerical simulation of Davies and $\mathrm{Li}$ [10] with a White-Metzner fluid in a journal bearing and, more recently, the numerical simulations of Shin et al. [11] using an explicit viscoelastic model which is much easier to solve. Other recent works on non-isothermal flow of viscoelastic fluids having temperature-dependent fluid properties are the simulations of Wachs and Clermont [12] and Marduel and Kunisch [13] in the 4:1 contraction benchmark flow. Whereas the former was basically aimed at assessing the performance of a numerical method under non-isothermal conditions, the latter developed a simple flow control strategy to reduce the size of recirculation zones. A point worth noting is that, in both previous references, first order schemes were used for the convective terms in the transport and constitutive equations and consequently severe numerical diffusion errors are bound to be present and especially so with viscoelastic fluids.

The present work is a contribution towards assessing and quantifying the extent of temperature-dependence fluid properties on the flow of viscoelastic fluids and simple corrective terms are proposed for heat transfer and friction factor correlations in some flows. This requires a realistic rheological constitutive equation as well as flows that are relevant in the perspective of polymer melt operations. The rheological model here considered is the Phan-Thien-Tanner (PTT) model [14] and the flows selected will be the fundamental developing and fully-developed channel flows which represent some of the typical conditions found in extrusion. The basic results for this investigation were obtained with an existing simulation method [15] which, for the present purposes, was extended to include solution of the energy equation. Naturally, it was necessary to validate these new developments against analytical solutions and for that purpose the literature was used $[6,7,16,17]$.

The paper is organised as follows: in the next section we present the relevant conservation equations and outline the numerical method used to solve them. To frame the objectives of this work, Section 3 briefly reviews procedures in the literature for correcting effects of temperature dependence of fluid properties on Nusselt number and friction factor correlations. In the ensuing results section, we start by validating the results of the non-isothermal viscoelastic simulations with data from the literature. Note that the validation of the present hydrodynamic computational method has been conducted in the past for isothermal Newtonian [18,19] and viscoelastic fluid flows [20,21]. The extensive investiga- 
tion of the non-isothermal developing channel flow with viscoelastic fluids will then be carried out to fully assess the extent of the temperature-dependent effects upon the flow and heat transfer characteristics. The results allow the quantification of recommended coefficients for correcting those effects but an alternative methodology is also proposed. The paper ends with a summary of the main conclusions.

\section{Equations and outline of solution procedure}

The equations to be solved for non-isothermal laminar flow of incompressible fluids are the equations of conservation of mass

$\frac{\partial u_{j}}{\partial x_{j}}=0$

linear momentum

$\frac{\partial\left(\rho u_{i}\right)}{\partial t}+\frac{\partial\left(\rho u_{j} u_{i}\right)}{\partial x_{j}}=-\frac{\partial p}{\partial x_{i}}+\frac{\partial \tau_{i j}}{\partial x_{j}}$,

and thermal energy

$\frac{\partial\left(\rho c_{p} T\right)}{\partial t}+\frac{\partial\left(\rho c_{p} u_{j} T\right)}{\partial \alpha_{j}}=\frac{\partial}{\partial x_{j}}\left(k \frac{\partial T}{\partial x_{j}}\right)+\tau_{i j} \frac{\partial u_{i}}{\partial x_{j}}$.

In these equations $u_{i}$ represents the velocity vector, $p$ the pressure, $\rho$ the density, $k$ the thermal conductivity, $c_{p}$ the specific heat, $T$ the temperature and $\tau_{i j}$ the stress tensor. Interaction between the velocity gradient tensor and the stress tensor is accounted for by the second term on the right-hand-side of Eq. (3). When the fluid is purely viscous this term represents the internal heat generation by viscous dissipation, while for elastic fluids it also includes stored elastic energy.

The stress field is given by a rheological constitutive equation and here we employ a simplified form of the PTT model (SPTT), given by

$$
\begin{aligned}
& Y\left(\tau_{k k}, T\right) \tau_{i j}+\lambda\left(\frac{\partial \tau_{i j}}{\partial t}+\frac{\partial\left(u_{k} \tau_{i j}\right)}{\partial x_{k}}\right) \\
& \quad=\eta\left(\frac{\partial u_{i}}{\partial x_{j}}+\frac{\partial u_{j}}{\partial x_{i}}\right)+\lambda\left(\tau_{j k} \frac{\partial u_{i}}{\partial x_{k}}+\tau_{i k} \frac{\partial u_{j}}{\partial x_{k}}\right),
\end{aligned}
$$

where $\lambda$ stands for the relaxation time and $\eta$ is the viscosity coefficient. The simplification invoked is that the parameter controlling the second normal stress difference is set to zero; this is a common simplification adopted in many works with the PTT model. The stresscoefficient function $Y$ is related to the rate of destruction of impermanent junctions in the molecular network and can be decoupled as

$Y\left(\tau_{k k}, T\right)=\phi(T) f\left(\tau_{k k}\right)$ where $\tau_{k k}$ is the trace of the stress tensor. Following Phan-Thien [22] the function $\phi(T)$ is arbitrarily set to unity at the reference temperature at which the material parameters of the model are determined and therefore thermal effects are not considered on the stress-coefficient function $Y$.

The stress-dependent part of the stress-coefficient function has the exponential form

$f\left(\tau_{k k}\right)=\exp \left(\frac{\varepsilon \lambda}{\eta} \tau_{k k}\right)$,

which can be linearised as:

$f\left(\tau_{k k}\right)=1+\frac{\varepsilon \lambda}{\eta} \tau_{k k}$

following the proposition of the original reference for the PTT [14].

This linearisation is adequate for small molecular deformations as occurs in weak flows, according to the flow classification of Tanner and Huilgol [23] (see also [24]). Steady channel, pipe and pure Couette flows belong to this category of weak flows for which $f\left(\tau_{k k}\right)$ can be described by Eq. (7). In strong flows the exponential form should be adopted because it provides the correct strain-thinning elongational viscosity behaviour at high strain rates. In this paper, we will employ the linearised form of Eq. (7) for the channel flow, because the flow is weak and for which analytical solutions are available.

For validation purposes, some calculations will also be carried out with inelastic non-Newtonian equations. For this simplified situation, the constitutive equation takes the form

$\tau_{i j}=\eta\left(\frac{\partial u_{i}}{\partial x_{j}}+\frac{\partial u_{j}}{\partial x_{i}}\right)$

The sets of governing equations (Eqs. (1)-(4)) are solved with the finite volume method described in detail in [15]. Basically, the solution domain is decomposed in many adjacent control volumes over which those equations are integrated and transformed into algebraic form. These discretized matrix equations are then solved sequentially, for each dependent variable $(u, p, \tau, T)$, with conjugate gradient solvers and the inherent nonlinearities due to the convection terms, the $f(\tau)$ term in the constitutive equations and the temperature dependence of properties are dealt with by iteration.

Regarding the accuracy of the calculations, the discretization of the various terms of Eqs. (2)-(4) was done by central differences, which is a second-order scheme. For the convective terms this was implemented via the deferred correction approach in combination with the upwind scheme to provide stability.

All calculations were carried out in a channel of halfwidth $H$ with fully-developed flow conditions at the inlet for velocity and stress, but with a constant inlet 
temperature $\left(T_{\mathrm{i}}\right)$. At the wall, the no-slip condition was imposed for velocity and stress and temperature $\left(T_{\mathrm{w}}\right)$ was set constant. Due to flow symmetry only half the geometry had to be considered and the corresponding symmetry boundary conditions were imposed to all quantities at the plane of symmetry.

\section{Corrections to constant property correlations to account for temperature effects}

In viscoelastic developing duct flows, the Nusselt number $N u$ and friction coefficient $C_{\mathrm{f}}$ can be expressed as functionals

$$
\begin{aligned}
N u & =f\left(\operatorname{RePr}, \varepsilon W e^{2}, B r, \frac{x}{L}\right) \quad \text { and } \\
C_{\mathrm{f}} & =f\left(\operatorname{RePr}, \varepsilon W e^{2}, B r, \frac{x}{L}\right),
\end{aligned}
$$

where the Nusselt number and friction coefficient are defined by

$N u \equiv \frac{h D_{\mathrm{H}}}{k} \quad$ and $\quad C_{\mathrm{f}} \equiv \frac{4 \tau_{\mathrm{w}}}{\frac{1}{2} \rho V^{2}}$

and the product of Reynolds and Prandtl numbers is usually referred to as a Péclet number, $P e \equiv R e P r$, and the Brinkman number $\mathrm{Br}$ measures the amount of viscous dissipation and is defined further down (Eq. (14)).

In Eq. (10) $D_{\mathrm{H}}$ is the hydraulic diameter, $\tau_{\mathrm{w}}$ is the wall shear stress and $h$ is the heat transfer coefficient that needs to be defined as a function of the wall heat flux $\dot{q}_{\mathrm{w}}$ and a suitable difference $\Delta T$. Here we consider $\Delta T=T_{\mathrm{w}}-T_{\mathrm{b}}$ where subscripts $\mathrm{w}$ and $\mathrm{b}$ designate wall and bulk values, respectively so that $\dot{q}=h \Delta T$. If these are local temperatures and $\dot{q}_{\mathrm{w}}$ is a local heat flux then $h$ and $N u$ represent the local heat transfer coefficient and Nusselt number, respectively. However, if $\dot{q}_{\mathrm{w}}$ is an average flux over a given length $L$ of duct, then $\Delta T$ should also be defined clearly and here we will use $\Delta T=\frac{T_{\mathrm{w} 1}+T_{\mathrm{w} 2}}{2}-\frac{T_{\mathrm{b} 1}+T_{\mathrm{b} 2}}{2}$ with subscripts 1 and 2 designating inlet and outlet, respectively. Similarly, the friction coefficient can also be local or average depending on whether $\tau_{\mathrm{w}}$ is a local or an average value. The hydraulic diameter is defined as usual [4] $\left(D_{\mathrm{H}} \equiv \frac{4 A}{P}\right.$, where $A$ is the duct cross-section area and $P$ its corresponding wetted perimeter).

The dependence of $N u$ and $C_{\mathrm{f}}$ on $x / L$ can be discarded for the case of fully-developed flow or flow around bodies. Since the fluid properties vary with temperature they have to be evaluated at a reference temperature.

When the variations in temperature are not too large it suffices to calculate the properties at the film temperature $T_{\mathrm{f}}$ which for internal flows is calculated by the following empiricism [25]
$T_{\mathrm{f}}=\frac{1}{4}\left(T_{\mathrm{w} 2}+T_{\mathrm{w} 1}+T_{\mathrm{b} 2}+T_{\mathrm{b} 1}\right)$.

If the temperature variations are large and the fluids are liquids a second correction, called the property ratio method (see [4, p. 276]) is required to correct the values of $N u$ and $C_{\mathrm{f}}$ given by Eq. (9), which are based on properties at constant temperature (subscript $\mathrm{CP}$ ). This correction to $\mathrm{Nu}$ and $C_{\mathrm{f}}$ should take into account the variation with temperature of all fluid properties, namely viscosity, thermal conductivity, heat capacity and, for viscoelastic fluids, the relaxation time. However, in practice this approach has been simplified following a purely empirical suggestion by Sieder and Tate [26] for the case of liquid flows whereby only the viscosity is assumed to vary. Their suggestion has been adopted by most authors [4,5,25,27], and can be expressed by

$\frac{N u}{N u_{\mathrm{CP}}}=\left(\frac{\eta_{\mathrm{w}}}{\eta_{\mathrm{b}}}\right)^{n}$,

$\frac{C_{\mathrm{f}}}{C_{\mathrm{f}, \mathrm{CP}}}=\left(\frac{\eta_{\mathrm{w}}}{\eta_{\mathrm{b}}}\right)^{m}$,

where the standard values of the exponents are $n=-0.14$ and $m=0.5$ for $\frac{\eta_{\mathrm{w}}}{\eta_{\mathrm{b}}}>1$ (cooling) and 0.58 for $\frac{\eta_{\mathrm{w}}}{\eta_{\mathrm{b}}}<1$ (heating).

In both equations, $\eta_{\mathrm{w}}$ is evaluated at $T_{\mathrm{w}}=\left(T_{\mathrm{w} 1}+\right.$ $\left.T_{\mathrm{w} 2}\right) / 2$ and $\eta_{\mathrm{b}}$ at $T_{\mathrm{b}}=\left(T_{\mathrm{b} 1}+T_{\mathrm{b} 2}\right) / 2$ but there is some disagreement regarding the temperature at which fluid properties are to be calculated for determining $N u_{\mathrm{CP}}$ and $C_{\mathrm{f}_{\mathrm{CP}}}$ with Eq. (9). Some authors [4,5,27] refer that the fluid properties should be calculated at the bulk temperature $T_{\mathrm{b}}$ whereas others [25,28] refer that the film temperature be used instead. Holman [28] even suggests that both temperatures can be used depending on the correlation. This is one of the issues studied in the present investigation. Nevertheless, and unless otherwise stated, fluid properties for the various non-dimensional numbers are evaluated at the film temperature.

Numerical values for the exponents $n$ and $m$ have been based on experiments and analysis for various fluids ranging from oil [26] to liquid metals (Deissler, according to [4]), but very few investigations have reported the corresponding behaviour for viscoelastic fluids. The issue is especially relevant because nonNewtonian fluids are often very viscous, and have large viscous dissipation effects so that even small temperature differences between the inlet and the wall can still result in large viscosity variations.

In what regards the heat transfer coefficient, Skelland [7] has given correlations for slurries obeying the Bingham plastic model and also for power law fluids. The property ratio method of Sieder-Tate was adopted to correct for temperature effects but with the viscosities substituted by parameters from the rheological equations, such as the consistency index $\left(K_{\mathrm{w}} / K_{\mathrm{b}}\right.$ instead 
of $\left.\eta_{\mathrm{w}} / \eta_{\mathrm{b}}\right)$. For high Reynolds number flows of nonNewtonian solutions in the laminar and turbulent regimes, Cho and Hartnett [29] confirmed the SiederTate corrections mentioned in the literature and introduced extra corrections to account for buoyancy effects on the Nusselt number and stated that there were no differences between inelastic and elastic fluids. However, it is known from $[1,16,17]$ that for highly viscoelastic fluids, such as polymer melts, the heat transfer characteristics are affected by fluid elasticity, especially in the presence of viscosity dissipation. Still, it is fair to say that work with non-Newtonian fluids is scarce and especially so for viscoelastic fluids under all flow conditions.

One of the main objectives of the present study is to demonstrate, based on numerical experiments, that the empirical property ratio method of Eqs. (12) and (13) can also be applied for viscoelastic fluids, specifically when these follow the PTT model, but that the exponents $m$ and $n$ should take different values and do not have a simple dependence on the dimensionless numbers (Brinkman number and $\varepsilon W e^{2}$ ). The issue of the reference temperature for calculating fluid properties to evaluate fluid properties in the property ratio method is also discussed.

\section{Results and discussion}

Prior to presenting new results, the present numerical methodology for viscoelastic fluids is verified and validated, with special attention on non-isothermal effects, which were absent from previous studies with the same methodology.

\subsection{Verification and validation}

We start by studying the thermal development of a Newtonian fluid in a channel with fully-developed hydrodynamic flow and negligible axial diffusion. For this case semi-analytical solutions are available as tables in Shah and London [27] and also as approximate expressions, for both channel and pipe flows for the two most common boundary conditions: constant wall temperature or heat flux. The solution depends on the normalised axial coordinate $x^{*} \equiv x /\left(D_{\mathrm{H}} P e\right)$. For moderate and large values of $x^{*}$ the solution to this SturmLiouville problem is well represented by a limited number of terms in the eigenfunction expansion (e.g. Shah and London used 121 terms for $x^{*}>10^{-4}$ ), but the accuracy deteriorates significantly for lower values of $x^{*}$ unless a very large number of terms is used. Then, a better alternative is to apply Levêque's approximation, which converges much faster when $x^{*} \rightarrow 0$. The results presented by Shah and London combine the best solutions from both methods.
Table 1

Characteristics of the four meshes used in the calculations of the thermal developing flow in a channel

\begin{tabular}{lrrl}
\hline Mesh & $N_{x}$ & $N_{y}$ & $f_{x}, f_{y}$ \\
\hline M1 & 272 & 20 & $1.0237,0.90$ \\
M2 & 272 & 40 & $1.0237,0.9497$ \\
M3 & 544 & 80 & $1.012,0.9747$ \\
M4 & 1088 & 120 & $1.006,0.9831$ \\
& & & \\
M3s & 272 & 80 & $1.0237,0.9747$ \\
M4s & 272 & 120 & $1.0237,0.9831$ \\
\hline
\end{tabular}

$N_{x}, N_{y}$-number of cells in longitudinal and transverse direction (from symmetry plane to wall). $f_{x}, f_{y}$-geometric expansion factor of cells in longitudinal and transverse direction.

After an initial study it was found that a slight mesh non-uniformity in the transverse direction, with finer computational cells near the wall, provided higher accuracy. Hence, the remaining computations used such non-uniform meshes, the characteristics of which are given in Table 1. The table shows that consistent mesh refinement was carried out for meshes M2, M3 and M4. The channel half-width $H=0.005 \mathrm{~m}$, but two different lengths were used: for problems without viscous dissipation $L=1 \mathrm{~m}(L /(2 H)=100)$, whereas a longer duct was required to attain fully-developed flow in the presence of viscous dissipation, $L=10 \mathrm{~m}(L /(2 H)=1000)$. Meshes M3s and M4s were used in calculations whenever axial diffusion was not neglected to allow speedier but equally accurate computations.

For the thermal flow development under fullydeveloped hydrodynamic inlet flow conditions, with constant wall temperature and no viscous dissipation (the Graetz-Nusselt problem for constant wall temperature) calculations were carried out with the two meshes M1 and M2 and the corresponding predictions of Nusselt number are compared with Shah and London [27] data in Fig. 1 ( $B r=0$ data). By definition, axial diffusion of thermal energy is neglected in the theoretical problem. Axial diffusion was also neglected in the calculations, except at the inlet plane because this would require significant changes to the code. In order to minimize the negative influence of inlet axial diffusion on the calculations, these were carried out for a high Prandtl number of 1000 and so, differences between theory and calculations were only limited to the very beginning of the channel (low values of $x^{*}$ ). The Reynolds number is defined with the hydraulic diameter as $R e \equiv \rho U 4 H / \eta$ and the Prandtl number is given by $P r \equiv \eta c_{p} / k$. It can be seen that for $x^{*}>10^{-4}$, predictions collapse onto Shah and London's [27] data within the accuracy of the calculations.

To quantify the accuracy of the calculations the four consecutively refined meshes of Table 1 were used to predict the Graetz-Nusselt problem in the presence of 


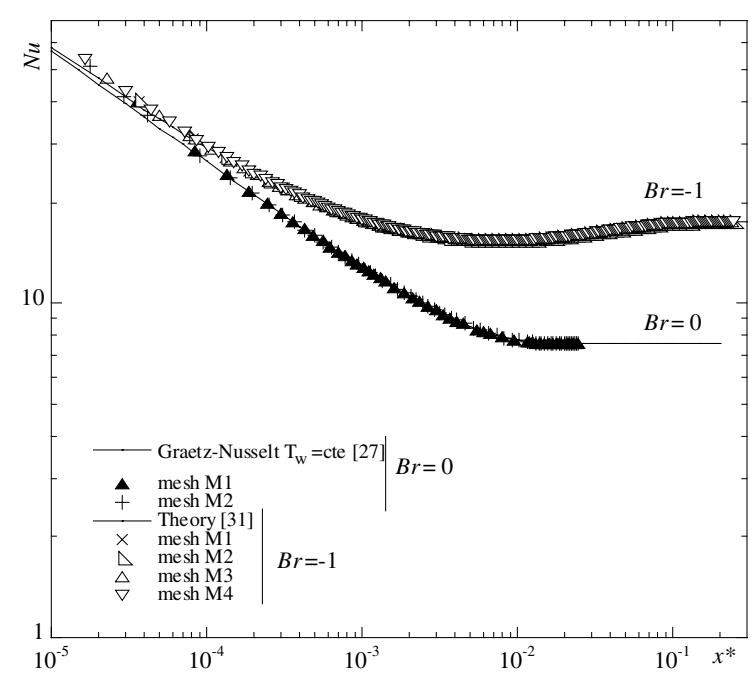

Fig. 1. Effect of mesh refinement on the longitudinal variation of Nusselt number for the Graetz-Nusselt flow of a Newtonian fluid in a channel $(R e=2, \operatorname{Pr}=1000)$ without $(B r=0)$ and with viscous dissipation $(B r=-1)$. (Only a quarter of the numerical data are plotted.)

viscous dissipation for both Newtonian and SPTT fluids. An assessment of the accuracy of those results under fully-developed conditions $\left(x^{*} \rightarrow \infty\right)$ is provided in Table 2, where calculated friction factors and Nusselt numbers are compared with results from the literature data for Newtonian and SPTT fluids. For the latter, those results were obtained by Coelho et al. [30], where the Graetz-Nusselt problem with the SPTT fluid was solved with a semi-analytical technique.

From data in both Tables the order of convergence of the numerical method was found to be equal to 2 in agreement with the formal second-order accuracy. The Nusselt numbers are closer to the theoretical value than the friction factors as would be anticipated from the high Prandtl number considered $(\operatorname{Pr}=1000)$.

The influence of mesh refinement on the predictions for the thermal development region can be assessed in Figs. $1(B r=-1)$ and 2 for Newtonian and SPTT fluids respectively, where the theoretical solutions for this

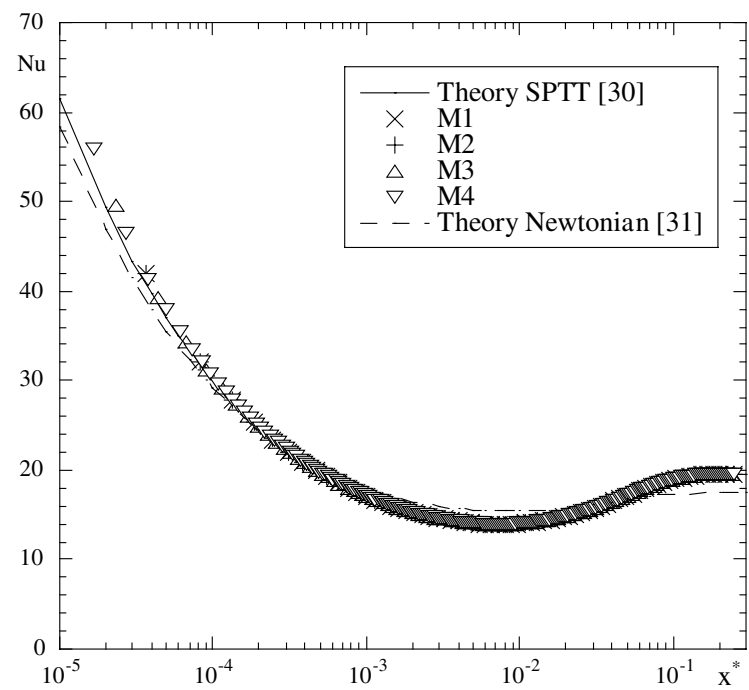

Fig. 2. Effect of mesh refinement on the longitudinal variation of the Nusselt number in Graetz-Nusselt channel flow with viscous dissipation $(B r=-1)$ for an SPTT fluid $\operatorname{Pr}=1000$, $R e=2, \varepsilon W e^{2}=0.1$. (Only a quarter of the numerical data are plotted.)

Graetz problem are also plotted (from [31,30], respectively). The fluid properties are considered independent of temperature but account is given to viscous dissipation $(B r=-1$, fluid cooling at wall) which is quantified by a non-dimensional Brinkman number defined as

$B r \equiv \frac{\eta U^{2}}{k\left(T_{\mathrm{w}}-T_{\mathrm{i}}\right)}$.

For the viscoelastic SPTT fluid, elasticity is measured by the product $\varepsilon W e^{2}$, where $W e$ is the Weissenberg number defined as $W e \equiv \lambda U / H$ and $\varepsilon$ is the extensibility parameter of the PTT model. These calculations were carried out for $\varepsilon W e^{2}=0.1$ and for the SPTT fluid the $N u$ data in Fig. 2 are about 5\% higher than for a Newtonian fluid represented by a dashed line. In the presence of viscous dissipation the effect of viscoelasticity on heat transfer is much enhanced, as documented in $[17,30]$. In both figures the agreement between predictions and theory is excellent.

Table 2

Effect of mesh refinement on computed friction factors and Nusselt number for fully-developed channel flow for constant wall temperature and $B r=-1\left(\varepsilon W e^{2}=0.1\right.$ for SPTT $)$

\begin{tabular}{|c|c|c|c|c|c|c|c|c|}
\hline \multirow[t]{2}{*}{ Mesh } & \multicolumn{4}{|c|}{ Newtonian } & \multicolumn{4}{|l|}{ SPTT } \\
\hline & $C_{\mathrm{f}}$ & Error $_{C_{\mathrm{f}}}$ & $N u$ & Error $_{N u}$ & $C_{\mathrm{f}}$ & Error $_{C_{\mathrm{f}}}$ & $N u$ & Error $_{N u}$ \\
\hline M1 & 47.870 & 0.271 & 17.4739 & 0.149 & 32.157 & 0.322 & 19.5716 & 0.204 \\
\hline M2 & 47.968 & 0.067 & 17.4935 & 0.037 & 32.239 & 0.069 & 19.6028 & 0.045 \\
\hline M3 & 47.992 & 0.016 & 17.4984 & 0.009 & 32.252 & 0.028 & 19.6094 & 0.011 \\
\hline M4 & 47.997 & 0.007 & 17.4993 & 0.004 & 32.256 & 0.017 & 19.6106 & 0.005 \\
\hline
\end{tabular}

Theoretical values: Newtonian fluids $-C_{\mathrm{f}}=48, N u=17.5$; SPTT fluid- $C_{\mathrm{f}}=32.261, N u=19.6116$. Error in [\%]. 
Although uncertainties of less than $0.1 \%$ are already obtained by using mesh M3, the effects we are looking for in this study require very accurate results of Nusselt number and friction factor, therefore mesh $\mathrm{M} 4 \mathrm{~s}$ is henceforth used in all calculations. Whenever a different calculation domain is required, a mesh having similar characteristics of fineness is used. Note that, in the remaining calculations, axial diffusion is always present. Therefore, the reasons for the discrepancies observed above at low values of $x^{*}$, other than those of numerical origin, disappear. Results from meshes M3s and M4s are not shown in Figs. 1 (for $B r=-1$ ) and 2 to avoid clutter, but they coincide with the results obtained with meshes M3 and M4.

\subsection{The effect of temperature-dependent properties}

These simulations were aimed at assessing the effect of temperature-dependent properties upon the heat transfer and hydrodynamic flow characteristics, and in particular to quantify such effect on the Nusselt number and friction coefficient. The properties of the fluid are characterized in Section 4.2.1.

The analysis proceeds in two parts: first, in Section 4.2.2 we look at the effect of temperature on each separate fluid property, and the consequences in terms of velocity and temperature profiles and of bulk flow characteristics of engineering interest (Nusselt number and friction factor). In addition, and since in a real flow all the properties are affected simultaneously, we also look at the combined effect when all properties are temperature dependent.

In the second part (Section 4.2.3), a parametric investigation on the effects of $\mathrm{Br}$ and $\varepsilon W e^{2}$ is carried out in an attempt to present a correction for the Nusselt number $N u$ and the friction coefficient $C_{\mathrm{f}}$ that takes into account the distortions in velocity and temperature profiles due to the variation of fluid properties with temperature. First, the film temperature is used to calculate fluid properties to be used in the calculations of $N u, C_{\mathrm{f}}, N u_{\mathrm{CP}}$ and $C_{\mathrm{f}, \mathrm{CP}}$ and in the more detailed discussion of the results. Then, in Section 4.2.4 the bulk temperature is also used in the calculations as reference temperature since its use is very frequent. Finally, a new correction method based on an equivalent temperature is presented in Section 4.2.5.

\subsubsection{Fluid properties}

The fluid properties whose dependence on temperature is investigated are the viscosity coefficient $\eta$ and the relaxation time $\lambda$ of the PTT model, the thermal conductivity $k$ and the heat capacity $c_{p}$. The variation with temperature of these properties is guided by data from actual polymer processing operations [32]. In some cases the fluid properties had to take values outside their scope for polymer melts in order to be able to reach some specific values of the non-dimensional numbers. We selected the following expressions to account for temperature variation of the fluid properties:

- For the viscosity $\eta$

$\eta(T)=\eta_{0} a_{T}$

with $\eta_{0}=10$ Pas (except $\eta_{0} \in[0.1,1]$ Pas for $B r \in[-1,1])$ and the shift factor $a_{T}$ given by the Arrhenius equation

$a_{T}=\exp \left[\alpha\left(\frac{1}{T+273.15}-\frac{1}{T_{0}+273.15}\right)\right]$

with $\alpha=1.72 \mathrm{E}+3 \mathrm{~K}$ and the reference temperature $T_{0}=190^{\circ} \mathrm{C}$;

- The relaxation time $\lambda$ follows a similar law

$\lambda(T)=\lambda_{0} a_{T}$

with $\lambda_{0}=0.002 \mathrm{~s}$;

- For the thermal conductivity $k$ a linear variation with temperature was used

$k(T)=k_{0}\left(k_{0}^{*}+k_{s}^{*} T\right)$

with $k_{0}=0.08 \mathrm{~W} /(\mathrm{mK}), k_{0}^{*}=0.7753$ and $k_{s}^{*}=0.00118$ $1 /{ }^{\circ} \mathrm{C}$;

- Finally, for the heat capacity $c_{p}$ again a linear variation was used as recommended by [32]

$c_{p}=c_{p, 0}\left(c_{p, 0}^{*}+c_{p, s}^{*} T\right)$

with $c_{p, 0}=0.4 \mathrm{~J} /(\mathrm{kgK}), \quad c_{p, 0}^{*}=1.2122$ and $c_{p, 2}^{*}=$ $-0.001121 /{ }^{\circ} \mathrm{C}$.

\subsubsection{The extent of the effect}

In order to grasp the magnitude of the effect of temperature-dependent properties on the variations of the velocity and temperature profiles and on the Nusselt number and friction factor, six simulations for developing channel flow (half-height $H=0.005 \mathrm{~m}$ ) were carried out in a $10 \mathrm{~m}$ long domain at $P e=\operatorname{Re} P r=100$, $\varepsilon W e^{2}=0.1, W e=0.4$ and $B r=-25$. These non-dimensional numbers were calculated with properties at the inlet temperature of $190{ }^{\circ} \mathrm{C}$ and the wall temperature was set constant at $185^{\circ} \mathrm{C}$ (giving a relative temperature variation of $\Delta T / T_{\mathrm{in}} \cong 1.1 \%$ ). In the reference simulation all properties were independent of temperature (subscript (C), in four simulations only one property depended on temperature and in the final simulation all properties were varied simultaneously. These calculations have in common the properties at inlet temperature, i.e., they are not compared on the basis of either the film temperature or the bulk temperature.

The normalised velocity profiles for fully-developed flow are compared in Fig. 3: the solid line is for constant properties (CP), the symbols pertain to a situation where only the viscosity or the relaxation time depended on 


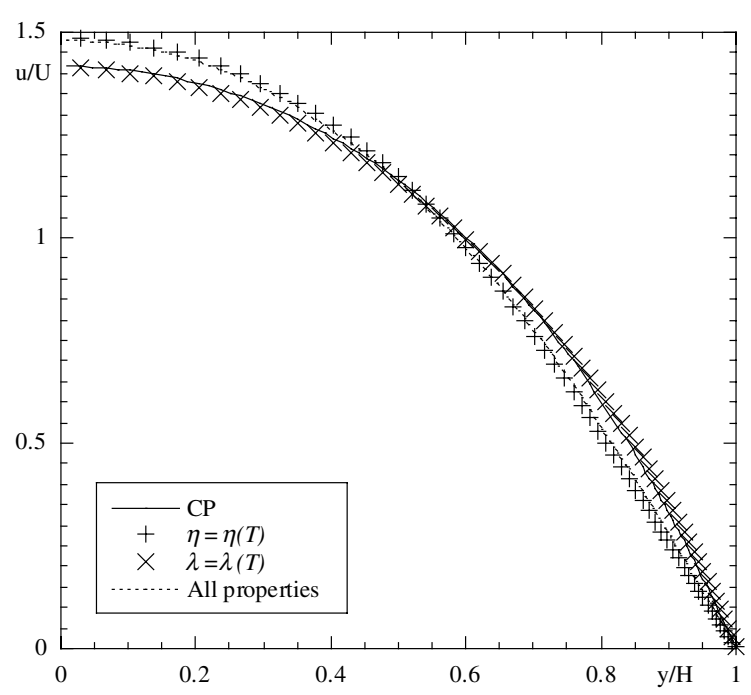

Fig. 3. Effect of temperature-dependent properties on normalised radial profile of velocity in a channel for an SPTT fluid in fully-developed flow $\left(\operatorname{RePr}=100, \quad \varepsilon W e^{2}=0.1, \quad W e=0.4\right.$, $B r=-25$. (Only half of the numerical data are plotted.)

temperature, and the dashed line is for the case when all properties were allowed to vary with temperature. Thermal properties can only affect the velocity indirectly via their effect upon the viscosity. Therefore, when the viscosity is assumed constant such indirect effect is switched off and the hydrodynamic problem remains decoupled from the thermal solution. The fluid property that has the largest influence on the flow kinematics is the viscosity, which raises the velocity in the center of the channel and decreases it near the wall and this is also apparent in the dashed line which is close to the curve for $\eta=\eta(T)$. The relaxation time has a small influence but of opposite sign.

This picture changes significantly when the normalised temperature profiles of Fig. 4 are analysed. The normalised temperature here used was defined as

$\theta \equiv \frac{T-T_{\mathrm{w}}}{T_{\mathrm{i}}-T_{\mathrm{w}}}$,

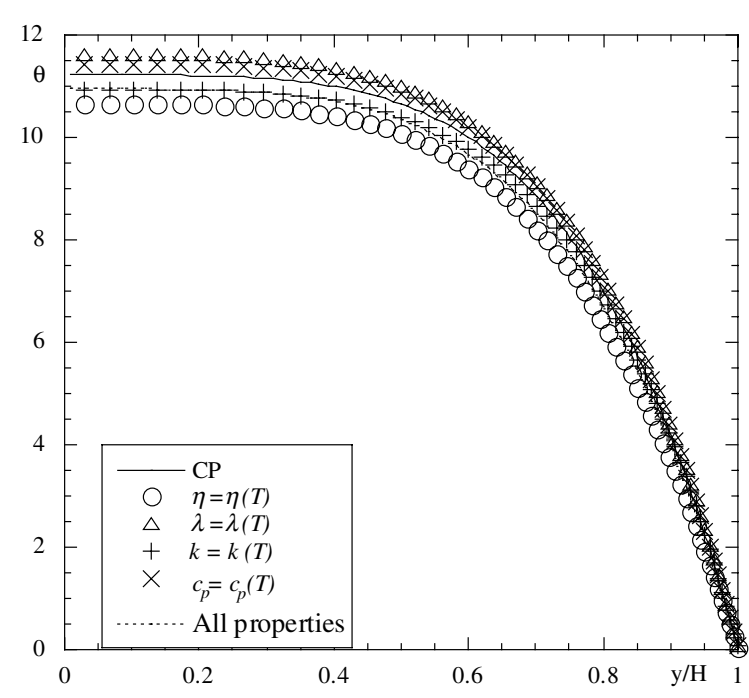

Fig. 4. Effect of temperature-dependent properties on the normalised radial profile of temperature $\theta=\left(T-T_{\mathrm{w}}\right) /\left(T_{\mathrm{i}}-T_{\mathrm{w}}\right)$ in a channel for an SPTT fluid in fully-developed flow $\left(\operatorname{Re} \operatorname{Pr}=100, \varepsilon W e^{2}=0.1, W e=0.4, B r=-25\right.$. (Only half of the numerical data are plotted.)

so that the true magnitude of the effects of the various properties can be assessed. Now, all properties seem important: thermal conductivity and heat capacity directly affect the temperature profile but so do the viscosity and relaxation time because of their effect on the velocity profile and the inclusion of viscous dissipation.

The effect of relaxation time on $\theta$, although small in the velocity profile, is now as large as the effect of viscosity alone, but again in the opposite direction. However, since these effects also affect bulk temperatures, we shall look next at bulk results which are of more engineering interest. Table 3 lists the values of the friction coefficient and Nusselt number corresponding to these six simulations and the corresponding relative variations due to temperature-dependent properties. The fluid properties at $190{ }^{\circ} \mathrm{C}$ (inlet temperature) were used to calculate all non-dimensional numbers. For the Nusselt number the Table lists both the value corresponding to

Table 3

Comparison between the friction factor and Nusselt number

\begin{tabular}{lllllll}
\hline Flow case & $N u$ & $\Delta N u / N u_{\mathrm{CP}}[\%]$ & $\overline{N u}$ & $\Delta \overline{N u} / \overline{N u}{ }_{\mathrm{CP}}[\%]$ & $C_{\mathrm{f}}$ & $\Delta C_{\mathrm{f}} / C_{\mathrm{f}, \mathrm{CP}}[\%]$ \\
\hline CP & 19.611 & - & 19.758 & - & 32.256 & - \\
$\eta=\eta(T)$ & 17.206 & -12.3 & 17.352 & -12.2 & 27.042 & -16.2 \\
$\lambda=\lambda(T)$ & 20.161 & +2.8 & 20.310 & +2.8 & 34.16 & +6.0 \\
$k=k(T)$ & 20.210 & +3.1 & 20.354 & +3.0 & 32.256 & - \\
$C_{p}=C_{p}(T)$ & 19.946 & +1.7 & 20.092 & +1.7 & 32.256 & - \\
All properties & 18.511 & -5.6 & 18.654 & -5.6 & 28.585 & -11.4 \\
\hline
\end{tabular}

Property variation with temperature followed Eqs. (15) to (19). Constant properties taken at $T_{\mathrm{i}}=190{ }^{\circ} \mathrm{C}$. 
fully-developed channel flow $(\mathrm{Nu})$ as well as the average value $(\overline{\mathrm{Nu}})$ encompassing the heat transfer in the whole duct from the entrance through the thermal developing region and into the fully-developed flow.

The variations in $N u$ and $\overline{N u}$ are very similar, so the analysis of the corrections in the next subsection basically needs to be carried out only for one of the two quantities. For convenience and to systematize we adopt the fully-developed values. The main effect comes from viscosity, which reduces $N u$ by $12 \%$ and $C_{\mathrm{f}}$ by $16 \%$ relative to the constant properties case. The effect of the relaxation time is to increase the friction coefficient by $6 \%$ (slightly more than a third of the effect of viscosity but in the opposite direction), an effect which is larger than suggested by Fig. 3. In contrast, the effect of the relaxation time on the Nusselt number is smaller than suggested by Fig. 4: $2.8 \%$ which is of the same order as the effect of heat capacity and thermal conductivity but, since these three smaller effects are in the same direction, their combination must be taken into account. Indeed, when all properties are allowed to vary with temperature the Nusselt number differs by only 5-6\% from the CP case, so the action of a temperature-dependent viscosity has been reduced by more than $50 \%$ by the combined effects of $k, \lambda$ and $c_{p}$.

We shall now present the results of a new set of simulations with a consistent choice of reference conditions, so as to allow an unambiguous quantification of the temperature effect, in agreement with the convention described in Section 3. In these simulations isothermal (CP) and non-isothermal cases have in common fluid properties calculated at the same film temperature and the corresponding results are presented in Table 4 . The exception to this are the last two runs, $\mathrm{i} 0 \mathrm{~b}$ and $\mathrm{i} 1 \mathrm{~b}$, where the fluid properties were calculated at the same bulk temperature.

Using the data in Table 4 and the property ratio correction method for each of the varying properties, values of the corresponding exponents can be calculated as follows

- From b0 and b1: $\frac{N u}{N u_{\mathrm{CP}}}=\left(\frac{\eta_{\mathrm{w}}}{\eta_{\mathrm{b}}}\right)^{n^{\prime}} \rightarrow n^{\prime}=-0.6253$; $\frac{C_{\mathrm{f}}}{C_{\mathrm{f}, \mathrm{CP}}}=\left(\frac{\eta_{\mathrm{w}}}{\eta_{\mathrm{b}}}\right)^{m^{\prime}} \rightarrow m^{\prime}=-0.5226$,

- $\mathrm{c} 0 \quad$ and $\quad \mathrm{c} 1: \quad \frac{N u}{N u_{\mathrm{CP}}}=\left(\frac{\lambda_{\mathrm{W}}}{\lambda_{\mathrm{b}}}\right)^{n^{\prime \prime}} \rightarrow n^{\prime \prime}=0.1556$; $\frac{C_{\mathrm{f}}}{C_{\mathrm{f}, \mathrm{CP}}}=\left(\frac{\lambda_{\mathrm{w}}}{\lambda_{\mathrm{b}}}\right)^{m^{\prime \prime}} \rightarrow m^{\prime \prime}=0.1282$,

- $\begin{aligned} & C_{\mathrm{f}, \mathrm{CP}} \\ & \mathrm{e} 0 \quad \text { and } \\ & \lambda_{\mathrm{b}}\end{aligned}$ $\frac{C_{\mathrm{f}}}{C_{\mathrm{f}, \mathrm{PP}}}=\left(\frac{k_{\mathrm{w}}}{k_{\mathrm{b}}}\right)^{m^{\prime \prime \prime}} \rightarrow m^{\prime \prime \prime}=0.0053$,

- $C_{\mathrm{f}, \mathrm{CP}} \quad\left(\frac{c_{\mathrm{b}}}{k_{\mathrm{b}}} \quad \mathrm{fl}\right.$ and $\quad \frac{N u}{N u_{\mathrm{CP}}}=\left(\frac{c_{p, \mathrm{w}}}{c_{p, \mathrm{~b}}}\right)^{n^{\prime \prime \prime \prime}} \rightarrow n^{\prime \prime \prime \prime}=0.5198 ;$
$\frac{C_{\mathrm{f}}}{C_{\mathrm{f}, \mathrm{CP}}}=\left(\frac{c_{p, \mathrm{w}}}{c_{p, \mathrm{~b}}}\right)^{m^{\prime \prime \prime \prime}} \rightarrow m^{\prime \prime \prime \prime}=-0.0052$.

When all properties vary the correction can be compounded, assuming independent contributions, according to

$\frac{N u}{N u_{\mathrm{CP}}}=\left(\frac{\eta_{\mathrm{w}}}{\eta_{\mathrm{b}}}\right)^{n^{\prime}}\left(\frac{\lambda_{\mathrm{w}}}{\lambda_{\mathrm{b}}}\right)^{n^{\prime \prime}}\left(\frac{k_{\mathrm{w}}}{k_{\mathrm{b}}}\right)^{n^{\prime \prime \prime}}\left(\frac{c_{p, \mathrm{w}}}{c_{p, \mathrm{~b}}}\right)^{n^{\prime \prime \prime \prime}}=0.9474$,

$\frac{C_{\mathrm{f}}}{C_{\mathrm{f}, \mathrm{CP}}}=\left(\frac{\eta_{\mathrm{w}}}{\eta_{\mathrm{b}}}\right)^{m^{\prime}}\left(\frac{\lambda_{\mathrm{W}}}{\lambda_{\mathrm{b}}}\right)^{m^{\prime \prime}}\left(\frac{k_{\mathrm{w}}}{k_{\mathrm{b}}}\right)^{m^{\prime \prime \prime}}\left(\frac{c_{p, \mathrm{w}}}{c_{p, \mathrm{~b}}}\right)^{m^{\prime \prime \prime \prime}}=0.9188$

with the same exponents as above. These results differ by $-0.29 \%$ and $-0.62 \%$ relative to the correct Nusselt number and friction coefficient of run i1, respectively when obtained from the corresponding $\mathrm{CP}$ values of run

Table 4

Comparison between the fully-developed friction factor and Nusselt number with properties calculated at film temperature, except i0b and ilb which use $T_{\mathrm{b}}$

\begin{tabular}{llllllll}
\hline Run & Property & $R e$ & $P r$ & $\varepsilon W e^{2}$ & $B r$ & $N u$ & $C_{\mathrm{f}}$ \\
\hline b0 & $\eta(T)$ & 2.139 & 46.75 & 0.1 & -23.38 & 17.2060 & 27.0418 \\
b1 & CP & 2.139 & 46.75 & 0.1 & -23.38 & 19.6116 & 30.1671 \\
c0 & $\lambda(T)$ & 2 & 50 & 0.0861 & -25 & 20.1609 & 34.1759 \\
c1 & CP & 2 & 50 & 0.0861 & -25 & 19.4718 & 33.2105 \\
e0 & $k(T)$ & 2 & 49.51 & 0.1 & -24.76 & 20.2104 & 32.2556 \\
e1 & CP & 2 & 49.51 & 0.1 & -24.76 & 19.6116 & 32.2610 \\
f0 & $c_{p}(T)$ & 2 & 49.45 & 0.1 & -25 & 19.9458 & 32.2556 \\
f1 & CP & 2 & 49.45 & 0.1 & -25 & 19.6116 & 32.2610 \\
i0 & All vary & 2.144 & 45.70 & 0.0870 & -23.09 & 18.5105 & 28.5850 \\
i1 & CP & 2.144 & 45.70 & 0.0870 & -23.09 & 19.4812 & 30.9188 \\
i0b & All vary & 2.379 & 39.90 & 0.0707 & -20.48 & 18.5105 & 28.5850 \\
i1b & CP & 2.379 & 39.90 & 0.0707 & -20.48 & 19.2919 & 28.9659 \\
\hline
\end{tabular}

$\operatorname{Re}=2, \operatorname{Pr}=50, \varepsilon W e^{2}=0.1$ and $B r=-25$ if properties calculated at $T_{\mathrm{i}}$. 
i0. These small differences are caused by the compensating effects of the various coupled phenomena.

From an engineering point of view, simpler expressions are more useful and the traditional way is to concentrate all the variations in a viscosity correction and determine a different exponent, i.e.,

- From i0 and i1: $\frac{N u}{N u_{\mathrm{CP}}}=\left(\frac{\eta_{\mathrm{w}}}{\eta_{\mathrm{b}}}\right)^{n} \rightarrow n=-0.2389 ; \frac{C_{\mathrm{f}}}{C_{\mathrm{f}, \mathrm{CP}}}=$ $\left(\frac{\eta_{\mathrm{w}}}{\eta_{\mathrm{b}}}\right)^{m} \rightarrow m=-0.3668$.

Exponents $n$ and $m$ differ significantly from those quoted in the literature (see Eqs. (12) and (13)), especially for the friction coefficient: we found a negative value $m=-0.37$ in contrast to the positive value of $m=0.5$ in Eq. (13). The exponent $n$ retained the same sign, but the difference is still large $(n=-0.14$ for Newtonian and $n=-0.24$ for PTT). If only the viscosity is allowed to depend on temperature (runs b0 and b1) the exponents $n^{\prime}$ and $m^{\prime}$ differ even more from those in the literature.

These values were obtained under the conditions quoted for Eqs. (12) and (13), namely $\eta_{\mathrm{w}}$ is evaluated at $\left(T_{\mathrm{w} 1}+T_{\mathrm{w} 2}\right) / 2$ and $\eta_{\mathrm{b}}$ at $\left(T_{\mathrm{b} 1}+T_{\mathrm{b} 2}\right) / 2$ and also with the fluid properties calculated at $T_{\mathrm{f}}$ in $N u, C_{\mathrm{f}}, N u_{\mathrm{CP}}$ and $C_{\mathrm{f}, \mathrm{CP}}$. Had we evaluated $\eta_{\mathrm{b}}$ at the average mixing temperature of the fluid within the channel (between the inlet and outlet), exponents $n$ and $m$ would have taken the values of -0.1381 and -0.2121 , respectively (for runs i0 and i1), i.e., $n$ would be very close to the value quoted in the literature but the sign of the friction coefficient exponent would still be negative. It can be argued that the discrepancy is due to the fact that the correct reference temperature for determining fluid properties is $T_{\mathrm{b}}$ rather than $T_{\mathrm{f}}$. Hence, we repeated cases i0 and i1 in Table 4 using the fluid properties at $T_{\mathrm{b}}$, now called $\mathrm{i} 0 \mathrm{~b}$ and $\mathrm{ilb}$, and the corresponding exponents were

- From i0b and i1b: $\frac{N u}{N u_{\mathrm{CP}}}=\left(\frac{\eta_{\mathrm{w}}}{\eta_{\mathrm{b}}}\right)^{n} \rightarrow n=-0.1933$; $\frac{C_{\mathrm{f}}}{C_{\mathrm{f}, \mathrm{CP}}}=\left(\frac{\eta_{\mathrm{w}}}{\eta_{\mathrm{b}}}\right)^{m} \rightarrow m=-0.0619$.

These two values are closer to those quoted in the literature (see Eqs. (12) and (13)) than those derived on the basis of $T_{\mathrm{f}}$ but the improvement is not dramatic and $m$ is still negative. Had we again evaluated $\eta_{\mathrm{b}}$ at the mixing temperature of the fluid rather than at $T_{\mathrm{b}}$ the values of the exponents would be $n=-0.1117$ and $m=-0.0358$, i.e., $n$ would now be worse than in the case of $T_{\mathrm{f}}$ which was very close to -0.14 .

In conclusion, the values of $n$ and $m$, and especially the latter, are very sensitive to the temperatures used to calculate the fluid properties, which must be defined unambiguously. Had we used the recommended exponents $n=-0.14$ and $m=0.5$ to calculate the true $N u$ and $C_{\mathrm{f}}$ from the corresponding temperature independent values $\left(N u_{\mathrm{CP}}\right.$ and $\left.C_{\mathrm{f}, \mathrm{CP}}\right)$ for the runs i0 and il in Table 4 , the errors would be $-2.1 \%$ and $+20.4 \%$ whereas for the runs $\mathrm{i} 0 \mathrm{~b}$ and $\mathrm{i} 1 \mathrm{~b}$ the same errors would be $+1.1 \%$ and $+12.8 \%$, respectively. If for these flow conditions the error in the Nusselt number is negligible for engineering purposes, the same is not true for the friction factor regardless of the temperature used to calculate fluid properties.

In the next subsection a detailed investigation is carried out to quantify $n$ and $m$ for the SPTT fluid in a channel flow as a function of $\mathrm{Br}$ and $\varepsilon W e^{2}$.

\subsubsection{Effect of $\mathrm{Br}$ and $\varepsilon W e^{2}$}

Fig. 5 shows the variation of the exponent $(n)$ required to correct the constant property Nusselt number $\left(N u_{\mathrm{CP}}\right)$, as a function of the elastic parameter $\varepsilon W e^{2}$ for different values of the Brinkman number. Note that $n$ was calculated on the basis of fully-developed conditions and $\varepsilon W e^{2}$ and $B r$ appearing in the graphs are based on properties calculated at the inlet temperature to reduce the number of computer simulations, because the film temperature is not known a priori. However, for each value of $B r$ and $\varepsilon W e^{2}$ the corresponding $N u$ and $N u_{\mathrm{CP}}$ are calculated with fluid properties determined at the same film temperature. If $\mathrm{Br}$ and $\varepsilon W e^{2}$ were to be calculated at $T_{\mathrm{f}}$ their values would vary from case to case. As shown in Fig. 5(a), there are basically two regions of behaviour: at high positive and negative Brinkman numbers, $n$ assumes small negative values, slowly varying with the Brinkman number; as $\mathrm{Br} \rightarrow 0, n$ varies strongly and even changes sign. The strongest variations are observed for positive Brinkman numbers $(B r>0$ is associated with $\left.T_{\mathrm{w}}>T_{\mathrm{i}}\right)$ and in the region where the critical Brinkman numbers are found. These critical Brinkman numbers correspond to changes in the direction of the wall heat flux and in the sign of the difference between bulk and wall temperatures due to the intense heat produced by viscous dissipation [17]. For a given $\varepsilon W e^{2}$ the critical Brinkman numbers will be different in the constant property and the temperature-dependent cases, leading to these large variations in $n$ and $m$, because the Nusselt number changes from high positive to high negative values or vice-versa.

Analysing these variations in more detail, Fig. 5(b) plots $n$ for $\mathrm{Br}<0$. At small negative Brinkman numbers, $n$ behaves monotonically with $\varepsilon W e^{2}$ and shows that the less elastic case has the more intense property correction ( $n=0$ means no correction). Similarly, $n$ should behave monotonically as $\mathrm{Br} \rightarrow-\infty$ with the less elastic case requiring the smallest correction, but this asymptotic behaviour has not yet been reached at $B r=-1000$ where $n=-0.263,-0.297,-0.293$ and -0.285 for $\varepsilon W e^{2}=0.1$, 1,10 and 100 , respectively. Viscous dissipation is seen to increase the required correction whereas elasticity slows down the variation of $n$ towards the asymptotic value.

For $\mathrm{Br}>0$, Fig. 5(c) shows the variation of $n$ and the situation here is rather more complex especially at low 

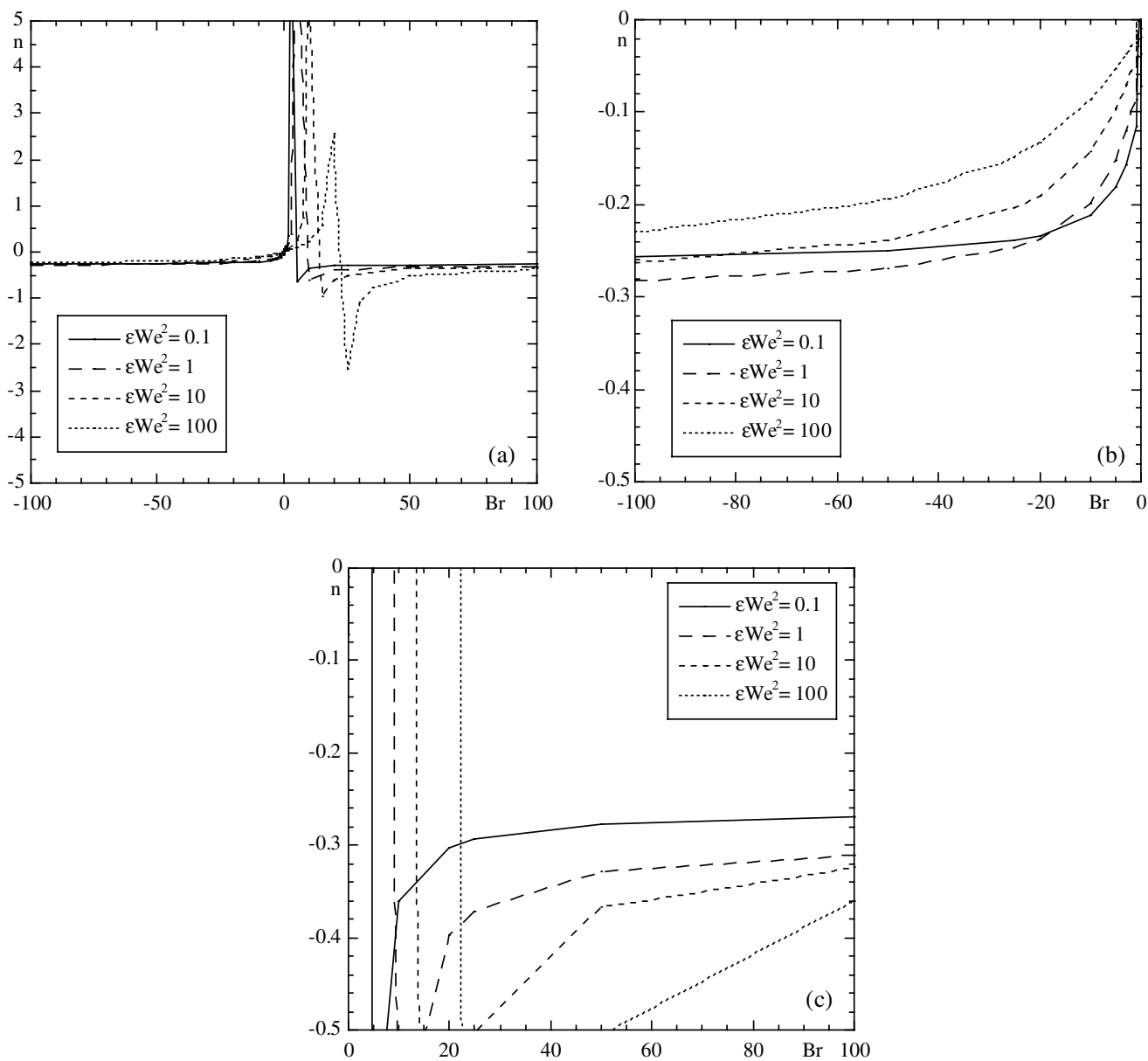

Fig. 5. Variation of exponent $n$ of Nusselt number correction with $B r$ and $\varepsilon W e^{2}$. Fluid properties were evaluated at the film temperature $\left(T_{\mathrm{f}}\right.$ ), but $B r$ and $\varepsilon W e^{2}$ in the abscissa were calculated at inlet temperature $\left(T_{\mathrm{i}}\right.$ ): (a) $-100<B r<100$; (b) zoom for $-100<B r<0$; (c) zoom for $0<B r<100$.

Brinkman numbers and for the reasons explained above. However, for $\mathrm{Br}$ larger than the critical Brinkman numbers, the variation of $n$ with $\varepsilon W e^{2}$ is always monotonic for all values of $\mathrm{Br}$ and, as for negative $\mathrm{Br}, n$ tends to asymptote for intense viscous dissipation, with a slower rate of variation of $n$ for the more elastic cases. The required correction increases with $\varepsilon W e^{2}$ as at high negative $B r$, but now, in contrast, the viscous dissipation reduces the correction.

In general, the value of $n$ is far from the quoted value of -0.14 for Newtonian fluids, but maintains the same sign. It is important at this stage to assess whether the use of $n=-0.14$ leads to large errors and Fig. 6 represents the error incurred by using the value of $n$ recommended in the literature for correcting $N u_{\mathrm{CP}}$ relative to the true $N u$, which is given by

Error $_{N u}=\left(\frac{\eta_{\mathrm{w}}}{\eta_{\mathrm{b}}}\right)^{-0.14-n}-1$.
In calculating Error $_{N u}$ the film temperature is being used to determine the fluid properties except for $\eta_{\mathrm{w}}$ and $\eta_{\mathrm{b}}$ determined at $T_{\mathrm{w}}$ and $T_{\mathrm{b}}$, respectively.

The errors are rather small, below $5 \%$ for $\mathrm{Br}<-0.1$ and $7 \%$ for $B r>0.1$ and decrease with parameter $\varepsilon W e^{2}$. In essence, and in spite of the differences found in $n$, the classical correction with $n=-0.14$ can still be used for engineering purposes because errors incurred are small, of the order of at most $2.5 \%$ for $|B r|>3$, except when viscous dissipation effects are weak. Under weak viscous dissipation conditions, and less so for fluids with high peaks of extensional viscosity $(\varepsilon \rightarrow 0)$, the errors can be significant and then it is recommended that the adequate corrections found in this work be used instead of the exponent value quoted in the literature.

For the friction coefficient corrective exponent $m$, Fig. 7 shows similar plots to those of $n$ in Fig. 5. For $B r<0$ the variation of $m$ in Fig. 7(b) is always monotonic with both $\varepsilon W e^{2}$ and $B r$ and $m$ also asymptotes 


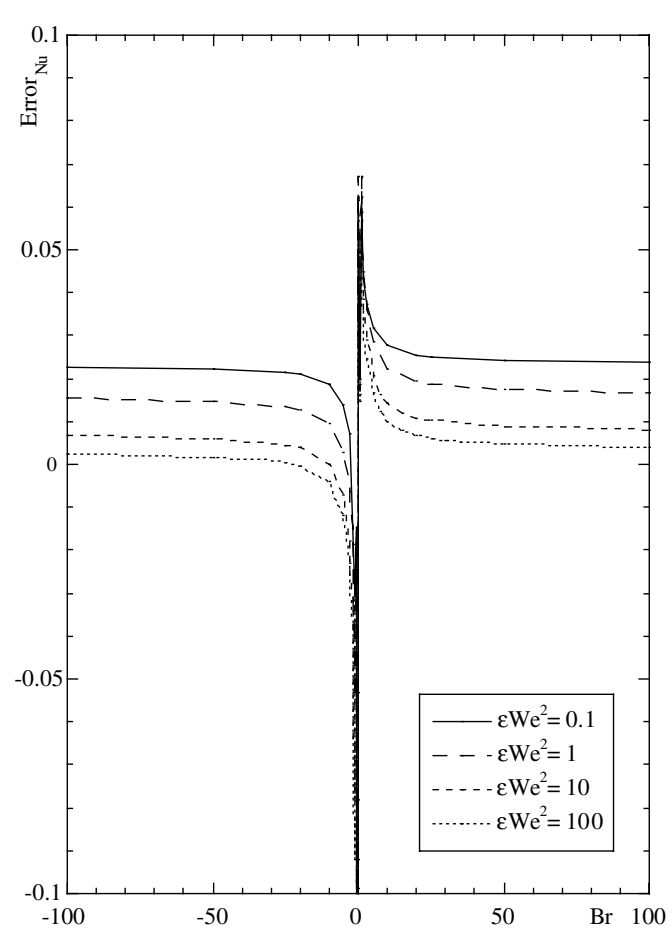

Fig. 6. Variation with $\mathrm{Br}$ and $\varepsilon W e^{2}$ of the error in Nusselt number associated with a $\mathrm{Nu}$ correction based on standard $n$ exponent when using $T_{\mathrm{f}}$ for calculating fluid properties.

for large negative values of $\mathrm{Br}$, with the corrections being smaller ( $n$ closer to 0 ) for the more elastic cases and increasing in all cases with viscous dissipation.

For $\mathrm{Br}>0$ plotted in Fig. 7(c), $m$ behaves monotonically with $B r$ but not with $\varepsilon W e^{2}$ except at very large values of $B r(B r>100)$. As with $n$, the corrections decrease with viscous dissipation. It may seem strange that $m$ shows the same type of variation as $n$, but this is due to the fact that the flow hydrodynamics and heat transfer are no longer decoupled when all fluid properties depend on temperature. Therefore, because the ratio $\eta_{\mathrm{w}} / \eta_{\mathrm{b}}$ reflects the variations in $T_{\mathrm{w}}$ and $T_{\mathrm{b}}$ associated with the passage of the critical Brinkman numbers, the exponent $m$ also behaves in a way similar to exponent $n$.

In general, and in contrast to $n$, the values of $m$ are very different from those quoted in the literature for Newtonian fluids ( 0.5 for cooling and 0.58 for heating), and are negative. As a consequence, as seen in Section 4.2.2, corrections to the friction coefficient are larger than those for the Nusselt number and cannot be neglected. The errors incurred in calculating $C_{\mathrm{f}}$ from the constant property $C_{\mathrm{f}, \mathrm{CP}}$ by using the recommended values of $m=0.5$ and 0.58 , and given by Eq. (24), are plotted in Fig. 8.

$$
\text { Error }_{C_{\mathrm{f}}}= \begin{cases}\left(\frac{\eta_{\mathrm{w}}}{\eta_{\mathrm{b}}}\right)^{0.50-m}-1 & \text { for } \frac{\eta_{\mathrm{w}}}{\eta_{\mathrm{b}}}>1, \\ \left(\frac{\eta_{\mathrm{w}}}{\eta_{\mathrm{b}}}\right)^{0.58-m}-1 & \text { for } \frac{\eta_{\mathrm{w}}}{\eta_{\mathrm{b}}}<1 .\end{cases}
$$

These errors are larger than Error $_{N u}$ but show the same pattern: constant Error $_{C_{\mathrm{f}}}$ for large positive and negative values of $B r$, increasing as viscous dissipation looses strength. However, the variation of Error $C_{\mathrm{f}}$ with $\varepsilon W e^{2}$ is more intense (for $\varepsilon W e^{2}=0.1$ there are errors of the order of $20 \%$ ) and as with Error $_{N u}$, a reduction in $\varepsilon W e^{2}$ increases the errors. Clearly, low values of $\varepsilon$ lead to large errors in predicting $C_{\mathrm{f}}$ with the standard exponents quoted in the literature. Here, in contrast to $N u$, the use of the correct coefficient is mandatory.

\subsubsection{What should be the reference temperature for fluid properties}

The issue of the adequate reference temperature to be used in calculating the fluid properties, mentioned at the start of Section 4.2, is discussed now. The investigation of the property ratio method in Section 4.2.3 was assessed using $T_{\mathrm{f}}$ and here we present the corresponding results obtained with $T_{\mathrm{b}}$ as the reference temperature for determining the fluid properties.

Fig. 9(a) plots the variation of $n$ with $B r$ and $\varepsilon W e^{2}$ for $B r<0$ corresponding to Fig. 5(b). As we can see the pattern is quite similar although the values of $n$ are now less negative than those of Fig. 9(b). However, this is not enough to explain the difference relative to the standard $n=-0.14$ value in the literature. For $B r>0$ Fig. 9(b) plots data that are comparable to the plots of Fig. 5(c) and here again the use of $T_{\mathrm{b}}$ provides less negative values of $n$ than the use of $T_{\mathrm{f}}$. That this is so can be checked in the plot of Error ${ }_{N u}$ in Fig. 10 which is directly comparable to Fig. 6. Except for weak viscous dissipation, Error ${ }_{N u}$ is lower than the corresponding quantity when using $T_{\mathrm{f}}$.

Regarding the friction factor corrective exponent $m$, the difference relative to the same exponent when using $T_{\mathrm{f}}$ is even more dramatic, but still insufficient to turn $m$ into a positive quantity as the standard literature value of $m$. Fig. 11 plots the variation of $m$ with $B r$ and $\varepsilon W e^{2}$ for $B r<0$ and is directly comparable with Fig. 7(b) whereas Fig. 12 represents the variation of Error $_{C_{\mathrm{f}}}$ with $B r$ and $\varepsilon W e^{2}$ and is directly comparable with Fig. 8. Whereas errors of the order of $20 \%$ are seen at $\varepsilon W e^{2}=0.1$ when using $T_{\mathrm{f}}$, these drop to around $13 \%$ when using $T_{\mathrm{b}}$ at large positive and negative values of $B r$. Since the use of $T_{\mathrm{b}}$ is more common than the use of $T_{\mathrm{f}}$, the values of $n$ and $m$ for the former are listed in Table 5.

In conclusion, the standard corrections for the Nusselt number and friction factor found in the literature, Eqs. (12) and (13), are not suitable to compensate for temperature effects for simple shear flows of viscoelastic fluids, with or without viscous dissipation. This disagreement is not caused by any ambiguity in defining the 

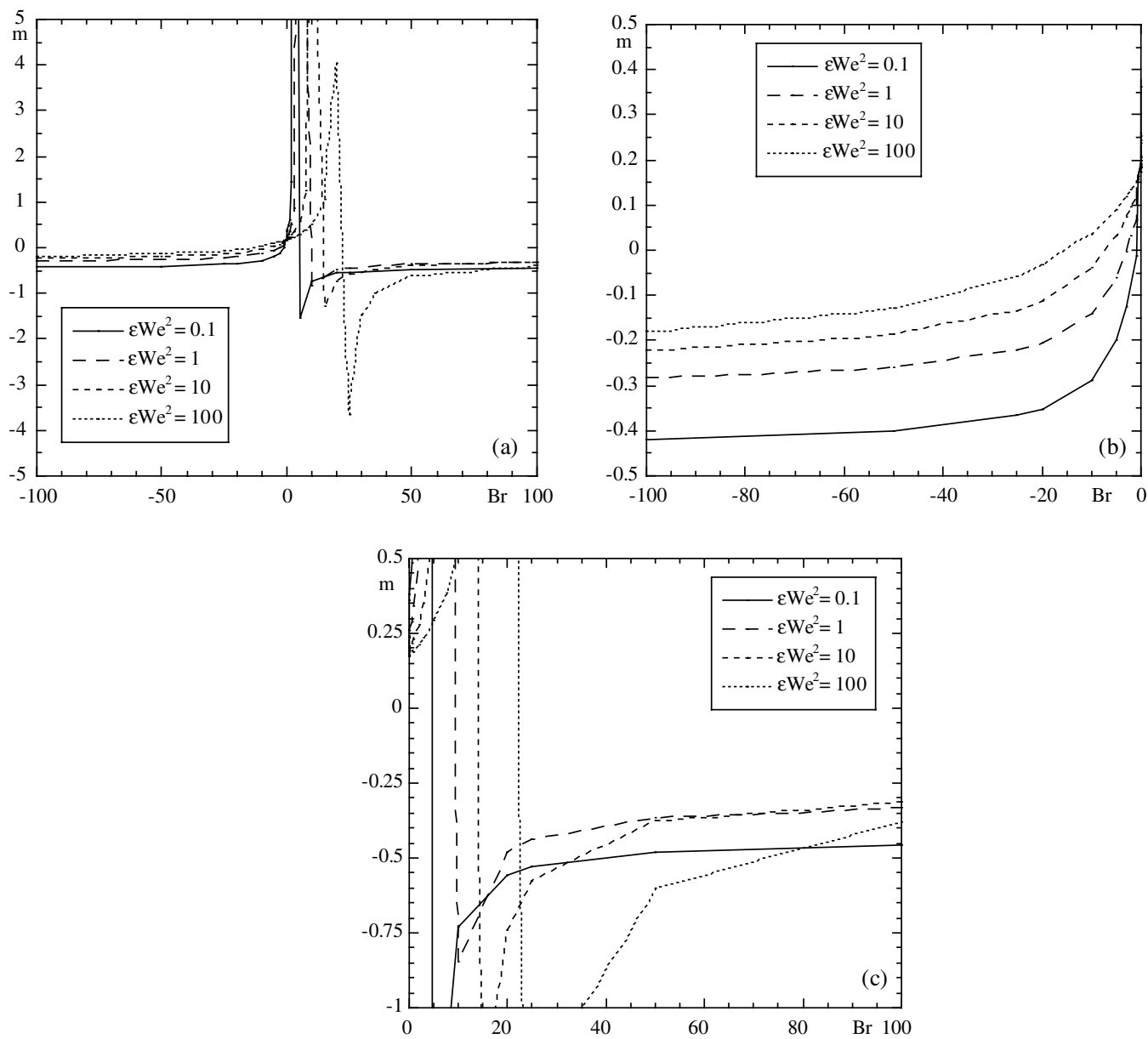

Fig. 7. Variation of exponent $m$ of friction factor correction with $B r$ and $\varepsilon W e^{2}$. Fluid properties were evaluated at the film temperature $\left(T_{\mathrm{f}}\right)$, but $\mathrm{Br}$ and $\varepsilon W e^{2}$ in the abscissa were calculated at inlet temperature $\left(T_{\mathrm{i}}\right)$ : (a) $-100<B r<100$; (b) zoom for $-100<B r<0$; (c) zoom for $0<B r<100$.

reference temperature used to calculate the fluid properties, although the errors are lower when using the bulk temperature $\left(T_{\mathrm{b}}\right)$ than with the film temperature $\left(T_{\mathrm{f}}\right)$.

\subsubsection{Equivalent temperature}

Regardless of the temperature used to quantify fluid properties, exponents $n$ and $m$ in the property ratio method vary significantly and in a complex way with $\mathrm{Br}$ and $\varepsilon W e^{2}$, especially in the vicinity of the condition for negligible viscous dissipation $(B r=0)$. This is caused by the various critical Brinkman numbers and the associated variations in the difference between bulk and wall temperatures and in the direction of the wall heat flux.

An alternative correction method for $N u$ and $C_{\mathrm{f}}$, that removes some of the variations seen with $n$ and $m$, is the use of an equivalent temperature $\left(T_{\mathrm{eq}}\right)$ to calculate fluid properties to be used in the Nusselt number and friction coefficient expressions for temperature independent properties. This correction was inspired by the methodology used to correct for temperature-dependent properties in high velocity gas flows where viscous dissipation is also important. There, a reference temperature, based on stagnation conditions, is used to calculate the fluid properties.

The determination of $T_{\text {eq }}$ was carried out by a numerical search method based on bracketing and bisection for the same fully-developed flow data and plots of the corresponding normalised equivalent temperature $\Delta T_{\text {eq }}^{*}$, to be used in the correction for $N u$ and $C_{\mathrm{f}}$ are shown in Figs. 13 and 14, respectively. Some of the data are listed in Table 6.

The normalised equivalent temperature is defined with the film temperature as

$\Delta T_{\mathrm{eq}}^{*} \equiv \frac{T_{\mathrm{eq}}-T_{\mathrm{f}}}{T_{\mathrm{f}}}$ 


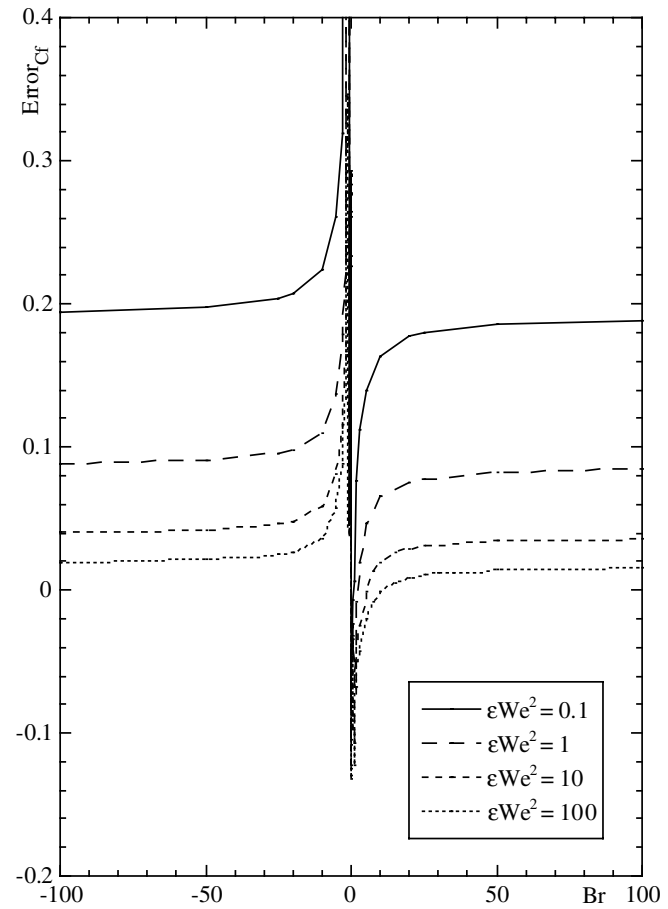

Fig. 8. Variation with $B r$ and $\varepsilon W e^{2}$ of the error in friction coefficient associated with a $C_{\mathrm{f}}$ correction based on standard $n$ exponent when using $T_{\mathrm{f}}$ for calculating fluid properties.

In this method to calculate the Nusselt number for fullydeveloped channel flow with constant wall temperature and viscous dissipation $(B r \neq 0)$, the fluid properties are determined at $T_{\mathrm{eq}}$ and expression (26), derived by Coelho et al. [17], is used. This takes into account the effect of temperature on fluid properties.

$N u=\frac{1925\left(1+\frac{6}{5} a\right)^{2}}{2\left(54 a^{2}+110 a+55\right)}$

with $a=9 \varepsilon W e^{2}\left(\frac{U_{N}}{U}\right)^{2}$ (see also [33]) and

$$
\begin{aligned}
& \frac{U_{N}}{U}=\frac{432^{1 / 6}\left(\delta^{2 / 3}-2^{2 / 3}\right)}{6 b^{1 / 2} \delta^{1 / 3}}, \\
& \delta=\left(3^{3} b+4\right)^{1 / 2}+3^{3 / 2} b^{1 / 2}, \\
& b=\frac{54}{5} \varepsilon W e^{2} .
\end{aligned}
$$

For $B r=0$ the expression for $N u$ is different and is given by the ratio of two long polynomials in $a$, Eq. (44) in Coelho et al. [17]. However, for $B r=0$ an equivalent temperature could not be determined not even if Eq. (26) is used. Thus, the equivalent temperature method for $\mathrm{Nu}$ fails for $B r=0$. This is so because at $B r=0$ the theoretical $N u$ is almost constant and it can not be changed by variations in fluid properties. Note that this feature is also present for Newtonian fluids and under these conditions only the property ratio method can be used.
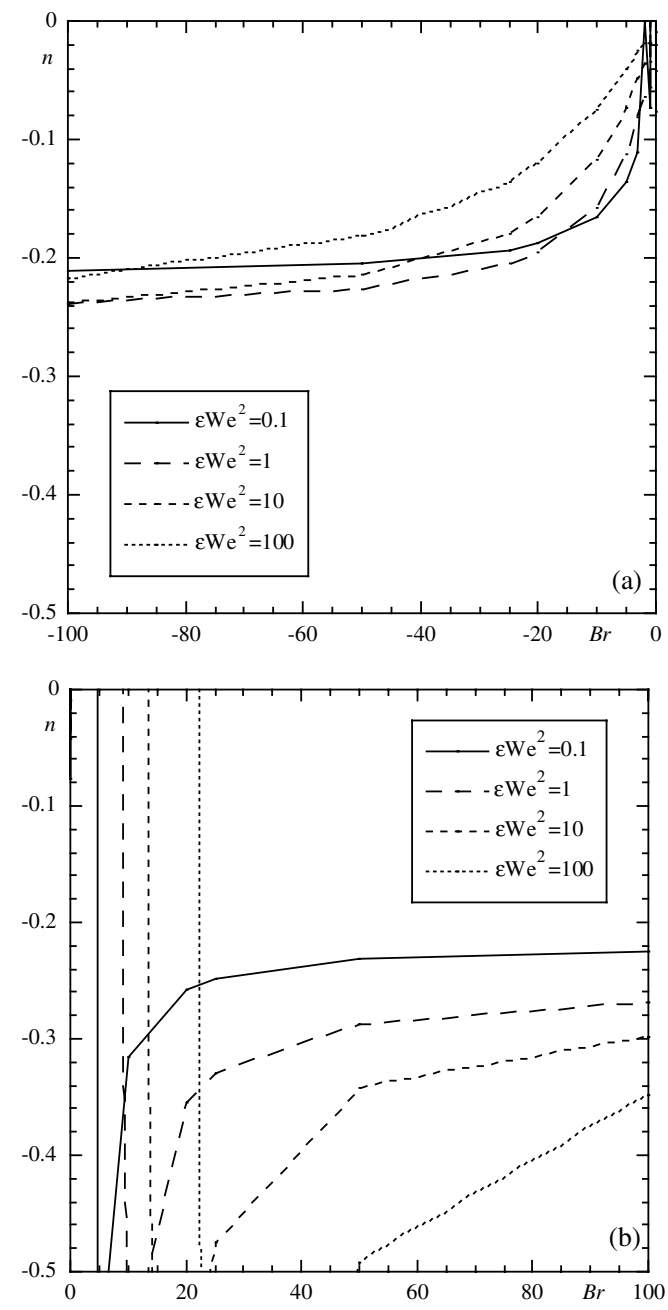

Fig. 9. Variation of exponent $n$ of Nusselt number correction with $B r$ and $\varepsilon W e^{2}$. Fluid properties were evaluated at the bulk temperature $\left(T_{\mathrm{b}}\right)$, but to calculate $\mathrm{Br}$ and $\varepsilon W e^{2}$ in the abscissa properties were calculated at inlet temperature $\left(T_{\mathrm{i}}\right)$ : (a) $-100<B r<100$; (b) $0<B r<100$.

The equivalent temperature for the friction factor is different and, as for the Nusselt number, it provides the temperature at which fluid properties must be determined to be used in the following equation for the friction factor in a channel flow (derived in [33])

$C_{\mathrm{f}}=\frac{96}{\operatorname{Re}} \frac{U_{N}}{U}$

with $U_{N} / U$ given above and the Reynolds number defined with the hydraulic diameter. In contrast to $T_{\mathrm{eq}, N u}$, $T_{\text {eq, } C_{\mathrm{f}}}$ can be defined for $B r=0$ because $C_{\mathrm{f}}$ is not given by a constant number.

Clearly, Figs. 13 and 14 show smoother and wellbehaved variations of $\left(\Delta T_{\mathrm{eq}}^{*}\right)_{N u}$ and $\left(\Delta T_{\mathrm{eq}}^{*}\right)_{C_{\mathrm{f}}}$ than those 


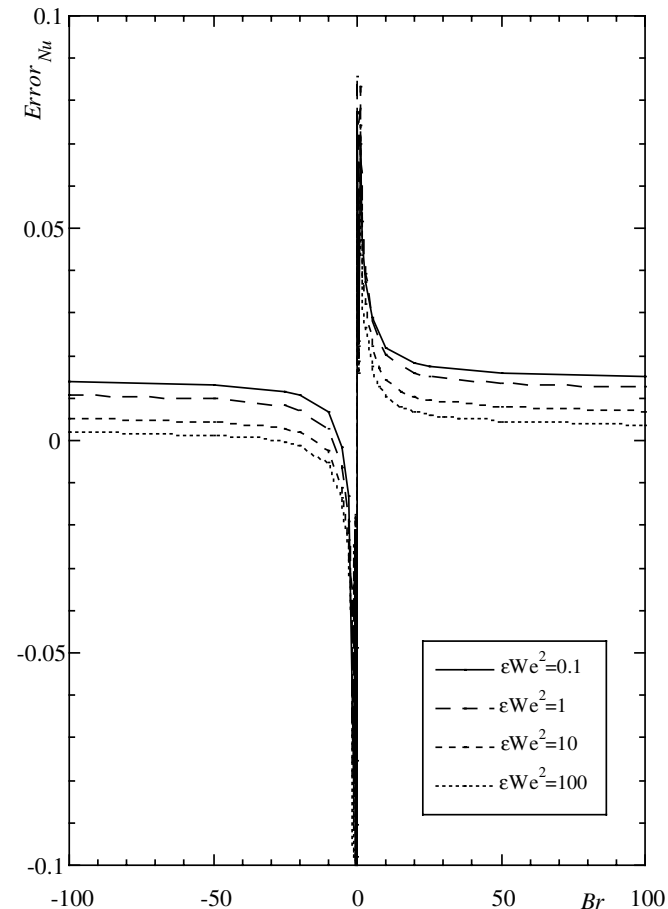

Fig. 10. Variation with $\mathrm{Br}$ and $\varepsilon W e^{2}$ of the error in Nusselt number associated with a $N u$ correction based on standard $n$ exponent when using $T_{\mathrm{b}}$ for calculating fluid properties.

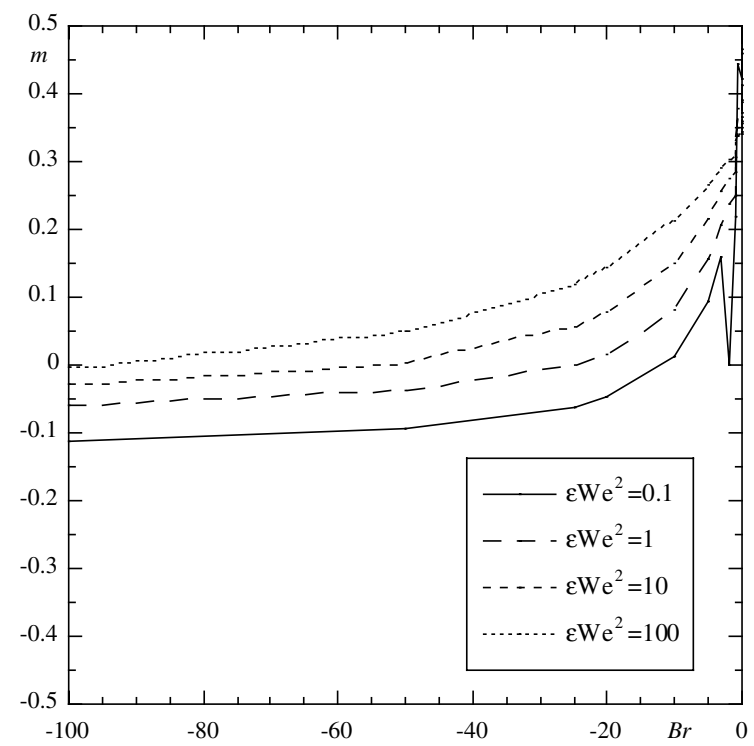

Fig. 11. Variation of exponent $m$ of friction factor correction with $B r$ and $\varepsilon W e^{2}$ for $-100<B r<0$. Fluid properties were evaluated at the bulk temperature $\left(T_{\mathrm{b}}\right)$, but $B r$ and $\varepsilon W e^{2}$ in the abscissa were calculated at inlet temperature $\left(T_{\mathrm{i}}\right)$.

of $n$ and $m$, except in the very near vicinity of $B r=0$. The asymptotic values at large positive and negative

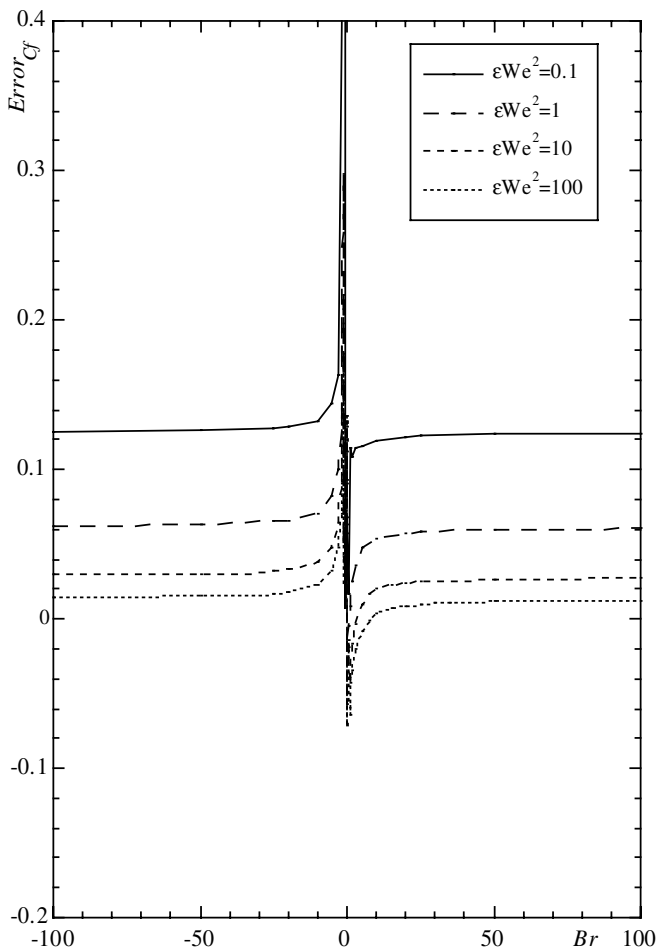

Fig. 12. Variation with $\mathrm{Br}$ and $\varepsilon W e^{2}$ of the error in friction factor associated with a $C_{\mathrm{f}}$ correction based on standard $m$ exponent when using $T_{\mathrm{b}}$ for calculating fluid properties.

Brinkman numbers are practically identical for $N u$ and $C_{\mathrm{f}}$ as can be assessed in the comparison of Fig. 15.

\section{Conclusions}

A finite volume calculation procedure was used to investigate the effect of temperature-dependent fluid properties on the friction factor and heat transfer in developing and fully-developed channel flow of viscoelastic fluids obeying a form of the PTT rheological constitutive equation with a linearised stress-coefficient function. It was shown that the use of the usual exponents (Eqs. (12) and (13)) in the property ratio method can result in large errors in predicting $N u$ and $C_{\mathrm{f}}$, especially when viscous dissipation is weak, and alternative values are presented as a function of the Brinkman number and a non-dimensionless number quantifying elastic effects, $\varepsilon W e^{2}$. However, the use of these values is rather complex as the problem is highly non-linear. The use of the bulk temperature to quantify $N u_{\mathrm{CP}}$ improved the values of $n$ and $m$ but the exponents were still too different from the standard coefficients.

An alternative method to account for the effects of temperature dependence of fluid properties on $N u$ and 
Table 5

Values of exponents $n$ and $m$ for correcting $N u$ and $C_{\mathrm{f}}$ using Eqs. (12) and (13) and fluid properties calculated at $T_{\mathrm{b}}$ for viscoelastic flows of PTT fluids in a channel with imposed constant wall temperature

\begin{tabular}{|c|c|c|c|c|c|c|c|c|}
\hline \multirow[t]{2}{*}{$B r$} & \multicolumn{2}{|c|}{$\varepsilon W e^{2}=0.1$} & \multicolumn{2}{|c|}{$\varepsilon W e^{2}=1$} & \multicolumn{2}{|c|}{$\varepsilon W e^{2}=10$} & \multicolumn{2}{|c|}{$\varepsilon W e^{2}=100$} \\
\hline & $n$ & $m$ & $n$ & $m$ & $n$ & $m$ & $n$ & $m$ \\
\hline-1000 & -0.217 & -0.128 & -0.252 & -0.079 & -0.262 & -0.066 & -0.259 & -0.059 \\
\hline-100 & -0.211 & -0.111 & -0.239 & -0.058 & -0.237 & -0.029 & -0.216 & -0.0032 \\
\hline-50 & -0.205 & -0.094 & -0.227 & -0.036 & -0.214 & +0.004 & -0.181 & +0.050 \\
\hline-25 & -0.193 & -0.062 & -0.205 & +0.0008 & -0.179 & +0.057 & -0.135 & +0.120 \\
\hline-10 & -0.166 & +0.013 & -0.157 & +0.081 & -0.118 & +0.149 & -0.075 & +0.212 \\
\hline-5 & -0.136 & +0.095 & -0.112 & +0.156 & -0.073 & +0.217 & -0.041 & +0.264 \\
\hline-3 & -0.111 & +0.158 & -0.082 & +0.207 & -0.048 & +0.255 & -0.025 & +0.290 \\
\hline-1 & -0.074 & +0.219 & -0.056 & +0.250 & -0.035 & +0.285 & -0.019 & +0.308 \\
\hline-0.5 & +0.019 & +0.443 & +0.037 & +0.379 & +0.027 & +0.351 & +0.016 & +0.338 \\
\hline-0.1 & +0.047 & +0.422 & +0.037 & +0.371 & +0.021 & +0.350 & +0.011 & +0.340 \\
\hline-0.01 & +0.083 & +0.461 & +0.053 & +0.388 & +0.028 & +0.358 & +0.014 & +0.344 \\
\hline 0 & -0.077 & +0.466 & -0.041 & +0.390 & -0.021 & +0.358 & -0.010 & +0.344 \\
\hline 0.01 & +0.075 & +0.742 & +0.097 & +0.518 & +0.067 & +0.412 & +0.030 & +0.367 \\
\hline 0.1 & +0.079 & +0.760 & +0.100 & +0.525 & +0.069 & +0.415 & +0.038 & +0.371 \\
\hline 0.5 & +0.169 & +0.804 & +0.124 & +0.522 & +0.069 & +0.414 & +0.034 & +0.373 \\
\hline 1 & +0.114 & +0.987 & +0.130 & +0.610 & +0.082 & +0.444 & +0.043 & +0.382 \\
\hline 3 & +5.72 & +17.7 & +0.441 & +1.11 & +0.153 & +0.550 & +0.064 & +0.418 \\
\hline 5 & -0.581 & -1.199 & +6.67 & +11.8 & +0.298 & +0.766 & +0.100 & +0.474 \\
\hline 10 & -0.316 & -0.415 & -0.508 & -0.616 & +5.75 & +8.89 & +0.248 & +0.698 \\
\hline 25 & -0.248 & -0.218 & -0.329 & -0.210 & -0.475 & -0.384 & -2.509 & -3.469 \\
\hline 50 & -0.232 & -0.171 & -0.287 & -0.138 & -0.342 & -0.185 & -0.493 & -0.421 \\
\hline 100 & -0.224 & -0.149 & -0.269 & -0.108 & -0.298 & -0.121 & -0.348 & -0.202 \\
\hline 1000 & -0.218 & -0.132 & -0.255 & -0.084 & -0.268 & -0.075 & -0.272 & -0.077 \\
\hline
\end{tabular}

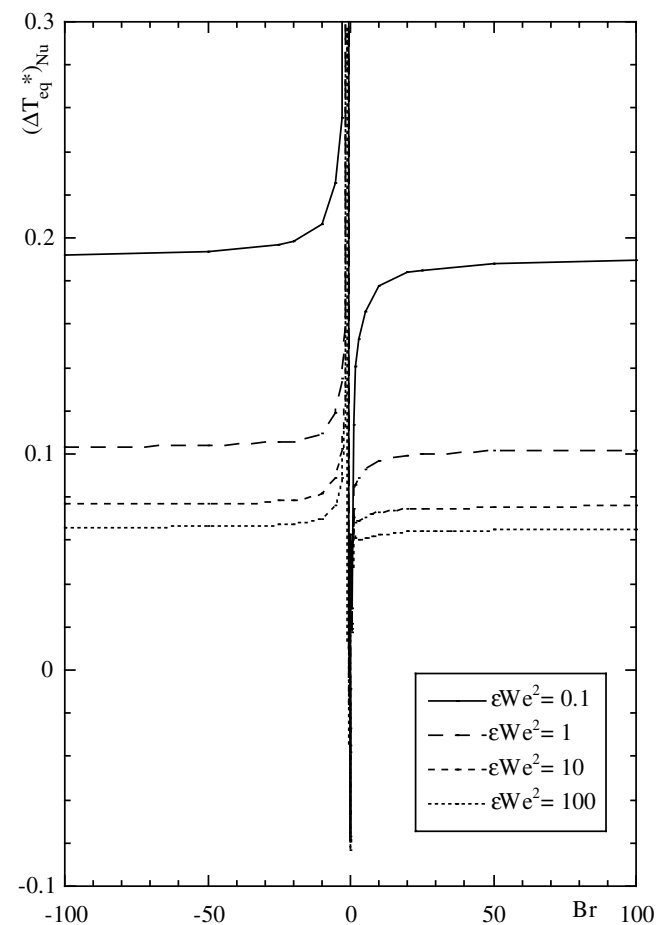

Fig. 13. Variation of the normalised equivalent temperature for the Nusselt number with $B r$ and $\varepsilon W e^{2} . B r$ and $\varepsilon W e^{2}$ calculated at $T_{\text {in }}$.

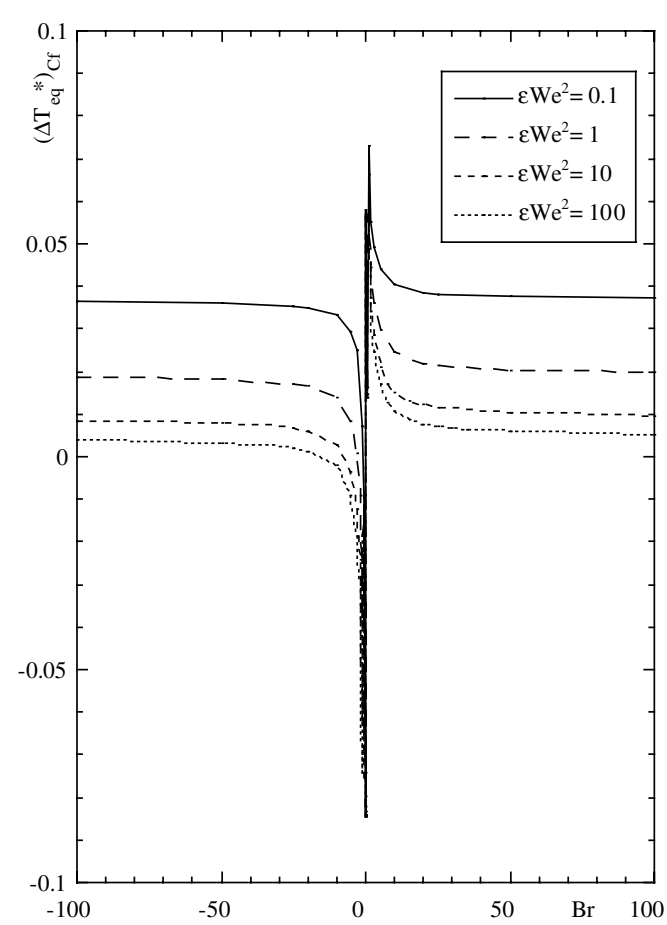

Fig. 14. Variation of the normalised equivalent temperature for the friction factor with $B r$ and $\varepsilon W e^{2} . B r$ and $\varepsilon W e^{2}$ calculated at $T_{\text {in }}$. 
Table 6

Values of $\left(\Delta T_{\mathrm{eq}}^{*}\right)_{N u}$ and $\left(\Delta T_{\mathrm{eq}}^{*}\right)_{C_{\mathrm{f}}}$ for calculating $N u$ and $C_{\mathrm{f}}$ using Eqs. (26) and (27) to account for temperature-dependent fluid properties for viscoelastic flows of PTT fluids in a channel with imposed constant wall temperature

\begin{tabular}{|c|c|c|c|c|c|c|c|c|}
\hline \multirow[t]{2}{*}{$B r$} & \multicolumn{2}{|c|}{$\varepsilon W e^{2}=0.1$} & \multicolumn{2}{|l|}{$\varepsilon W e^{2}=1$} & \multicolumn{2}{|l|}{$\varepsilon W e^{2}=10$} & \multicolumn{2}{|c|}{$\varepsilon W e^{2}=100$} \\
\hline & $\left(\Delta T_{\text {eq }}^{*}\right)_{N u}$ & $\left(\Delta T_{\text {eq }}^{*}\right)_{C_{\mathrm{f}}}$ & $\left(\Delta T_{\mathrm{eq}}^{*}\right)_{N u}$ & $\left(\Delta T_{\mathrm{eq}}^{*}\right)_{C_{\mathrm{f}}}$ & $\left(\Delta T_{\text {eq }}^{*}\right)_{N u}$ & $\left(\Delta T_{\mathrm{eq}}^{*}\right)_{C_{\mathrm{f}}}$ & $\left(\Delta T_{\mathrm{eq}}^{*}\right)_{N u}$ & $\left(\Delta T_{\mathrm{eq}}^{*}\right)_{C_{\mathrm{f}}}$ \\
\hline-1000 & +0.191 & +0.037 & +0.103 & +0.019 & +0.077 & +0.0091 & +0.065 & +0.0042 \\
\hline-100 & +0.192 & +0.036 & +0.103 & +0.019 & +0.077 & +0.0085 & +0.066 & +0.0039 \\
\hline-50 & +0.194 & +0.036 & +0.104 & +0.018 & +0.077 & +0.0079 & +0.066 & +0.0032 \\
\hline-25 & +0.197 & +0.035 & +0.105 & +0.017 & +0.078 & +0.0067 & +0.067 & +0.0019 \\
\hline-10 & +0.207 & +0.033 & +0.110 & +0.014 & +0.082 & +0.0029 & +0.070 & -0.0021 \\
\hline-5 & +0.225 & +0.030 & +0.119 & +0.008 & +0.089 & -0.0035 & +0.076 & -0.0089 \\
\hline-3 & +0.256 & +0.025 & +0.135 & +0.001 & +0.102 & -0.0124 & +0.089 & -0.0185 \\
\hline-1 & +0.655 & +0.007 & +0.348 & -0.039 & +0.309 & -0.0629 & +0.297 & -0.0743 \\
\hline-0.5 & +0.010 & -0.009 & 0.000 & -0.012 & -0.003 & -0.0128 & -0.005 & -0.0132 \\
\hline-0.1 & -0.009 & -0.074 & -0.027 & -0.080 & -0.035 & -0.0822 & -0.038 & -0.0833 \\
\hline-0.01 & -0.077 & -0.083 & -0.078 & -0.084 & -0.080 & -0.0842 & -0.083 & -0.0842 \\
\hline 0 & & -0.085 & & -0.084 & & -0.0844 & & -0.0843 \\
\hline 0.01 & +0.057 & +0.056 & +0.057 & +0.057 & +0.055 & +0.0564 & +0.034 & +0.0558 \\
\hline 0.1 & +0.063 & +0.058 & +0.059 & +0.057 & +0.058 & +0.0570 & +0.057 & +0.0569 \\
\hline 0.5 & +0.029 & +0.016 & +0.022 & +0.015 & +0.019 & +0.0141 & +0.018 & +0.0137 \\
\hline 1 & +0.114 & +0.073 & +0.085 & +0.066 & +0.075 & +0.0612 & +0.070 & +0.0589 \\
\hline 3 & +0.153 & +0.049 & +0.089 & +0.036 & +0.069 & +0.0284 & +0.060 & +0.0248 \\
\hline 5 & +0.166 & +0.044 & +0.093 & +0.030 & +0.071 & +0.0210 & +0.061 & +0.0170 \\
\hline 10 & +0.178 & +0.041 & +0.097 & +0.024 & +0.073 & +0.0152 & +0.063 & +0.0109 \\
\hline 25 & +0.185 & +0.038 & +0.100 & +0.021 & +0.075 & +0.0116 & +0.064 & +0.0071 \\
\hline 50 & +0.188 & +0.038 & +0.101 & +0.020 & +0.076 & +0.0104 & +0.065 & +0.0058 \\
\hline 100 & +0.189 & +0.037 & +0.102 & +0.020 & +0.076 & +0.0098 & +0.065 & +0.0052 \\
\hline 1000 & +0.191 & +0.037 & +0.103 & +0.019 & +0.077 & +0.0092 & +0.065 & +0.0043 \\
\hline
\end{tabular}

$C_{\mathrm{f}}$, determines an equivalent temperature to calculate properties to be used in the theoretical expressions for constant properties. Values of this normalised equiva-

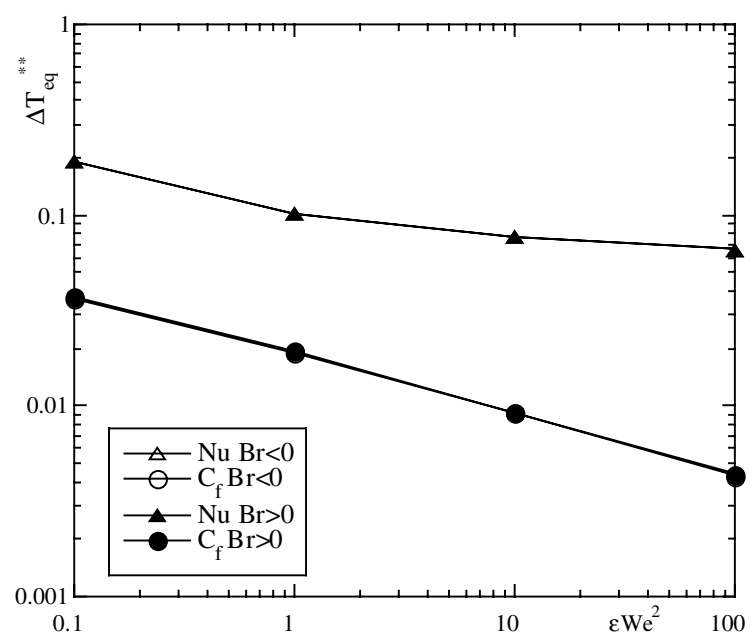

Fig. 15. Variation with $\varepsilon W e^{2}$ of the asymptotic values of the normalised equivalent temperatures. $\varepsilon W e^{2}$ in abscissa calculated at $T_{\text {in }}$. lent temperature were quantified and the method was seen to be a better alternative than the property ratio method, except under weak viscous dissipation conditions where it still did not provide a simple correction technique.

For the steady simple shear flow considered in this study it could be argued that an alternative and simpler way to arrive at the same results would be to prescribe an adequate viscosity function, $\eta$, and solve the relevant governing equations as in the case of the generalised Newtonian fluid. It is important to realize that such approach is not feasible because in the present problem the motion, the constitutive, and the energy equations are coupled through a temperature-dependent-property effect and no simple expression for the viscosity coefficient, in terms of $\dot{\gamma}$ and $T$, can be derived. We are therefore faced with the problem of solving the full set of equations for velocity, stress and temperature, as done in this work.

\section{Acknowledgements}

The authors greatfully acknowledge funding by FCT, Fundação para a Ciência e Tecnologia, under the POCTI and Plurianual programmes. 


\section{References}

[1] J.-F. Agassant, P. Avenas, J.P. Sergent, P.J. Carreau, Polymer Processing: Principles and Modelling, Hansers Publishing, New York, 1991.

[2] R.B. Bird, R.C. Armstrong, O. Hassager, Dynamics of polymeric liquids, in: Fluid Mechanics, 2nd ed., vol. 1, John Wiley and Sons, New York, 1987.

[3] F.C. Chou, C.W. Tung, The mechanism of heat transfer enhancement for mineral oil in 2:1 rectangular ducts, Int. J. Heat Mass Transfer 38 (1995) 2863-2871.

[4] W.M. Kays, M.E. Crawford, Convected Heat and Mass Transfer, 2nd ed., McGraw-Hill, New York, 1980.

[5] E.R.G. Eckert, R.M. Drake Jr., Analysis of Heat and Mass Transfer, McGraw-Hill, New York, 1972.

[6] G. Forrest, W.L. Wilkinson, Laminar heat transfer to power law fluids in tubes with constant wall temperature, Trans. Inst. Chem. Eng. 51 (1973) 331-338.

[7] A.H.P. Skelland, Non-Newtonian Flow and Heat Transfer, John Wiley and Sons, New York, 1967.

[8] J.M. Kenny, J.M. Castro, N.J. Capiati, Analysis of the wall slip effect in thermoplastic polymer extrusion, Lat. Am. J. Chem. Eng. Appl. Chem. 15 (1985) 59-75.

[9] S. Shin, Y.I. Cho, Laminar heat transfer in a rectangular duct with a non-Newtonian fluid with temperature-dependent viscosity, Int. J. Heat Mass Transfer 37 (1994) 19-30.

[10] A.R. Davies, X.K. Li, Numerical modelling of pressure and temperature effects in viscoelastic flow between eccentrically rotating cylinders, J. Non-Newton Fluid Mech. 54 (1994) 331-350.

[11] S. Shin, H.-H. Ahn, Y.I. Cho, C.-H. Sohn, Heat transfer behavior of a temperature-dependent non-Newtonian fluid with Reiner-Rivlin model in a 2:1 rectangular duct, Int. J. Heat Mass Transfer 42 (1999) 2935-2942.

[12] A. Wachs, J.-R. Clermont, Non-isothermal viscoelastic flow computations in an axisymmetric contraction at high Weissenberg numbers by a finite volume method, J. NonNewton Fluid Mech. 95 (2000) 147-184.

[13] X. Marduel, K. Kunisch, Suboptimal control of transient non-isothermal viscoelastic fluid flows, Phys. Fluids 13 (2001) 2478-2491.

[14] N. Phan-Thien, R.I. Tanner, A new constitutive equation derived from network theory, J. Non-Newton Fluid Mech. 2 (1977) 353-365.

[15] P.J. Oliveira, F.T. Pinho, G.A. Pinto, Numerical simulation of non-linear elastic flows with a general collocated finite-volume method, J. Non-Newton Fluid Mech. 79 (1998) 1-43.

[16] F.T. Pinho, P.J. Oliveira, Analysis of forced convection in pipes and channels with the simplified Phan-Thien-Tanner, Int. J. Heat Mass Transfer 43 (2000) 2273-2287.
[17] P.M. Coelho, F.T. Pinho, P.J. Oliveira, Fully-developed forced convection of the Phan-Thien-Tanner fluid in ducts with a constant wall temperature, Int. J. Heat Mass Transfer 45 (2001) 1413-1423.

[18] P.J. Oliveira, F.T. Pinho, Pressure drop coefficient of laminar Newtonian flow in axisymmetric sudden expansions, Int. J. Heat Fluid Flow 18 (1997) 518-529.

[19] M.P. Escudier, I. Gouldson, P.J. Oliveira, F.T. Pinho, Effects of inner cylinder rotation on laminar flow of a Newtonian fluid through an eccentric annulus, Int. J. Heat Fluid Flow 21 (2000) 92-103.

[20] M.A. Alves, F.T. Pinho, P.J. Oliveira, Effect of a highresolution differencing scheme on finite-volume predictions of viscoelastic flows, J. Non-Newton Fluid Mech. 93 (2000) 287-314.

[21] M.A. Alves, F.T. Pinho, P.J. Oliveira, The flow of viscoelastic fluids past a cylinder: finite volume high resolution methods, J. Non-Newton Fluid Mech. 97 (2001) 205-230.

[22] N. Phan-Thien, A nonlinear network viscoelastic model, J. Rheol. 22 (1978) 259-283.

[23] R.I. Tanner, R.R. Huilgol, Rheol. Acta 14 (1975) 959962.

[24] R.I. Tanner, Engineering Rheology, 1st ed., Clarendon Press, 1985.

[25] R.B. Bird, W.E. Stewart, E.N. Lightfoot, Transport Phenomena, 2nd ed., 2002.

[26] E.N. Sieder, C.E. Tate, Heat transfer and pressure drop of liquids in tubes, Ind. Eng. Chem. 28 (1936) 1429.

[27] R.K. Shah, A.L. London, Laminar Flow Forced Convection in Ducts, Academic Press, New York, 1978.

[28] J.P. Holman, Heat Transfer, 5th ed., McGraw-Hill, 1981.

[29] Y.I. Cho, J.P. Hartnett, Non-Newtonian fluids, in: W.M. Rohsenow, J.P. Hartnett, E.N. Ganic (Eds.), Handbook of Heat Transfer Applications, 2nd ed., 1985 (Chapter 2).

[30] P.M. Coelho, F.T. Pinho, P.J. Oliveira, Thermal entry flow for a viscoelastic fluid: the Graetz problem for the PTT model, Int. J. Heat Mass Transfer 46 (20) (2003) 38653880 .

[31] J.W. Ou, K.C. Cheng, Viscous dissipation effects on thermal entrance heat transfer in laminar and turbulent pipe flows with uniform wall temperature, ASME paper no. 74-HT-50, presented at the AIAA/ASME 1974 Thermophysics and Heat Transfer Conference, Boston, Massachusetts, US, 1974.

[32] J.E. Mark, Physical Properties of Polymers Handbook, American Institute of Physics, New York, 1996.

[33] P.J. Oliveira, F.T. Pinho, Analytical solution for fully developed channel and pipe flow of Phan-Thien-Tanner fluids, J. Fluid Mech. 387 (1999) 271-280. 